\title{
AFFERENT CONNECTIONS OF DORSAL AND VENTRAL AGRANULAR INSULAR CORTEX IN THE HAMSTER MESOCRICETUS AURATUS
}

\author{
R. L. REEP and S. S. WINANS* \\ Department of Anatomy, The University of Michigan Medical School, Ann Arbor, MI 48109, U.S.A.
}

\begin{abstract}
The agranular insular cortex is transitional in location and structure between the ventrally adjacent olfactory allocortex primutivus and dorsally adjacent sensory-motor isocortex. Its ventral anterior division receives major afferent projections from olfactory areas of the limbic system (posterior primary olfactory cortex, posterolateral cortical amygdaloid nucleus and lateral entorhinal cortex) while its dorsal anterior division does so from non-olfactory limbic areas (lateral and basolateral amygdaloid nuclei).

The medial segment of the mediodorsal thalamic nucleus projects to both the ventral and dorsal divisions of the agranular insular cortex, to the former from its anterior portion and to the latter from its posterior portion. Other thalamic inputs to the two divisions arise from the gelatinosus, central medial, rhomboid and parafascicular nuclei. The dorsal division, but not the ventral division, receives input from neurons in the lateral hypothalamus and posterior hypothalamus.

The medial frontal cortex projects topographically and bilaterally upon both ventral and dorsal anterior insular cortex, to the former from the ventrally located medial orbital and infralimbic areas, to the latter from the dorsally-located anterior cingulate and medial precentral areas, and to both from the intermediately located prelimbic area. Similarly, the ipsilateral posterior agranular insular cortex and perirhinal cortex project in a topographic manner upon the two divisions of the agranular insular cortex.

Commissural input to both divisions originates from pyramidal neurons in the respective contralateral homotopical cortical area. In each case, pyramidal neurons in layer $\mathrm{V}$ contribute $90 \%$ of this projection and $10 \%$ arises from layer 111 pyramidals.

In the brainstem, the dorsal raphe nucleus projects to the ventral and dorsal divisions of the agranular insular cortex and the parabrachial nucleus projects to the dorsal division.

Based on their cytoarchitecture, pattern of afferent connections and known functional properties, we consider the ventral and dorsal divisions of the agranular insular cortex to be, respectively, periallocortical and proisocortical portions of the limbic cortex.
\end{abstract}

The existence of an insular cortex in rodents and other non-primate mammals was first made clear by Maximilian Rose in cytoarchitectural and developmental studies on several orders of mammals. ${ }^{51.52} \mathrm{He}$ described agranular and granular divisions of the lissencephalic rodent cortex which correspond to cortical areas of similar location and structure in the opercularized insulae of primates including humans. Through his examination of normal embryological material, Rose found that in mice, rabbits and humans the insular cortex and underlying claustrum both appear to develop from cortical and striatal elements that migrate ventrally to form a common cell plate (his cell strip X). He concluded that the insular cortex and claustrum are unique entities, distinct in origin and structure from both isocortex and striatum.

* Author to whom requests for reprints should be sent.

Abbreviations: Al, agranular insular cortex; AId/AIp/ AIv, dorsal/posterior/ventral agranular insular cortex; HRP, horseradish peroxidase; MD, mediodorsal thalamic nucleus.
In more recent comparative cytoarchitectonic and embryological studies, Sanides ${ }^{54}$ and others ${ }^{1,2,16,61.68}$ have emphasized the central position of agranular insular cortex in a structural transition from the relatively few layers of ventrally adjacent allocortex primitivus (olfactory cortex) to the distinct six layers of dorsally adjacent isocortex. Furthermore, agranular insular cortex is but one portion of a belt of agranular cortex that also includes orbital frontal and medial frontal transition cortical areas. ${ }^{35}$ This belt may represent a phylogenetic growth ring of the cortex, as suggested by Sanides. ${ }^{54}$

Several recent anatomical studies have demonstrated the striking parallels in afferent and efferent connections which exist among the insular, orbital and medial frontal transition cortical areas. A prime example is the presence, in several orders, of convergent input from the mediodorsal nucleus of the thalamus, basolateral nucleus of the amygdala and dopaminergic cells of ventral tegmental area, in both the agranular insular and medial frontal cortical areas. ${ }^{3,12,35,36}$

Comparative functional investigations on rodents, 
carnivores and primates have revealed that all regions of the frontal agranular transition cortex influence motivated and social behaviors ${ }^{15,31,32,33,56,59}$ as well as visceromotor and somatomotor responses usually associated with emotion and arousal for a review, see $\mathrm{Kaada}^{28}$ ). Such functions are often discussed collectively within the conceptual framework of the term 'limbic system'. Historically, the use of this term (see White ${ }^{65}$ ) in reference to cortical areas has emphasized medial zones, particularly the cingulate gyrus, to such an extent that the anatomical and functional continuity among the medial, orbital and insular areas is not commonly appreciated.

The rodent agranular insular cortex consists of several cytoarchitectural divisions, the exact number depending on the species. ${ }^{52}$ These divisions are known to differ with respect to certain of their afferent connections, as was found by Krettek \& Price in more recent autoradiographic studies of the cortical projections of the thalamus and amygdala in the rat. ${ }^{35,36}$ Furthermore, these divisions are likely to have differing functional influences which should become clearer as more specific lesion/behavior studies are done. For these reasons, we began studies of the afferent and efferent connections of the dorsal and ventral agranular insular areas (AId and AIv), which are the subdivisions of anterior agranular insular cortex in hamsters and rats. The efferent projections are treated in a forthcoming report. ${ }^{49}$

In a recent horseradish peroxidase (HRP) study in the rat, Gerfen \& Clavier ${ }^{17}$ reported differences in the thalamic inputs to AId and AIv, but did not find differences between AId and Alv with respect to other inputs. Some of the known inputs to Ald and AIv were not described at all. The account below, which gives the results of our HRP study of Ald and AIV, reveals many previously undescribed differences in the pattern of inputs to these two cortical areas and presents data concerning the cell types of origin, laminar distribution and topographic organization of particular projections.

\section{EXPERIMENTAL PROCEDURES}

In thirty male golden hamsters (Mesocricetus auratus) ranging in weight from 95.145 grams, iontophoretic deposits of horseradish peroxidase (Miles Laboratories, Inc.. Elkhart, Indiana), were made in the agranular insular cortex or surrounding areas. HRP was prepared at a concentration of $500 \mu \mathrm{g} / \mu \mathrm{l}$ in $0.05 \mathrm{M}$ Tris buffer (ph 8.6). The hamster to be used was anesthetized with sodium pentobarbital $(75 \mathrm{mg} / \mathrm{kg})$ and placed in a Kopf stereotaxic holder. A circular flap of skull bone was removed and the dura beneath incised. A glass monofilament micropipette (A-M Systems, Inc., Everett, WA) having a tip diameter of $12-24 \mu \mathrm{m}$, and containing the HRP solution, was lowered to the chosen coordinate position. A negative constant DC holding current of $0.1-0.3$ microamps was applied during the descent, from a Grass \$44 stimulator. This was to minimize leakage of HRP from the pipette tip. For depositing the HRP, positive DC pulses of $0.5-2,0$ microamps (one per second, $500 \mathrm{~ms}$ duration) were applied. After $515 \mathrm{~min}$ the current was turned off and the pipette left in place for up to $10 \mathrm{~min}$. During withdrawal of the pipette the negative holding current was again used.

Thirty to fifty hours after surgery, the animal was reanesthetized and perfused through the heart with $250 \mathrm{ml}$ of warm $(37 \mathrm{C})$ cacodylate-buffered saline solution (with $0.25 \mathrm{~g}$ sodium nitrite added as a vasodilator) followed by $250 \mathrm{ml}$ of cacodylate-buffered $3^{\prime \prime}$, glutaraldehyde fixative. The brain was removed from the skull and soaked overnight in cold $\left(4 \mathrm{C}\right.$ ) cacodylate-buffered saline with $25^{\circ}$ sucrose added. The brain was then cut coronally at $4050 \mu \mathrm{m}$ thickness on a freezing microtome and the sections col. fected and stored in a cold (on ice) solution consisting of $25 \%$ sucrose in ethylene glycol. From a few hours to a few days after cutting, every fourth section was reacted for HRP using tetramethylbenzidine as the chromagen and the procedures of de Olmos, Hardy \& Heimer, "The reacted sections were mounted from cold $(4 \mathrm{C})$ water onto glass slides and after having air-dried for several hours. were quickly dipped in alcohol and xylene then overlaid with coverslips. The distribution of labelled cells was examined using dark-field illumination, and plotted on tracings of adjacent Cresyl Violet stained sections which had been made using a Bausch and Lomb microprojector, and upon which relevant cytoarchitectural boundaries had been delineated.

\section{RESULTS}

\section{Technical note}

As noted by Newman \& Winans, ${ }^{43}$ an HRP deposit site can be parcelled into three distinct zones. When viewed with dark-field optics, these appear as: (1) a central area in which cells and the extracellular space are densely filled with HRP reaction product; (2) a middle zone in which cells are densely filled but the extracellular space only moderately so; (3) an outer halo of lightly-labelled cell bodies and no extracellular label. As discussed by these authors, retrograde transport apparently occurs only from zones 1 and 2. Thus in the present report all deposit site ligures represent zones 1 and 2 , and all references to the 'deposit site' refer to zones 1 and 2 .

Labelled profiles were considered to be neurons only if there was a clearly demarcated cell body containing HRP reaction product. Often, dendritic branches containing label could be seen emanating from the cell body as well. Terminal fields labelled by anterograde transport of HRP were visible in much of our material but will be considered in a forthcoming report on the efferent connections of AId and Alv. ${ }^{49}$

\section{Cytoarchitecture of agranular insular cortex}

As shown in Fig. 1A, the agranular insular cortex (AI) lies just dorsal to the rhinal fissure and extends from near the frontal pole anteriorly to perirhinal cor tex posteriorly. Throughout its length, AI is positioned between the ventrally adjacent primary olfactory cortex (terminology of Heimer ${ }^{22}$ ) and dorsally adjacent sensory-motor isocortex. Congruent with the 
early cytoarchitectural study of Rose $\mathrm{R}^{52}$ on several orders of mammals, including the mouse and squirrel as representative rodents, and the more recent cytoarchitectural and experimental studies of Krettek \& Price $^{35,36}$ in the rat, the hamster AI can be divided into anterior and posterior regions, and the anterior region subdivided into dorsal and ventral zones (AId and AIv), on both cytoarchitectural and connectionistic grounds. Area AIv curves around the buried fundus of the rhinal fissure and is thus not wholly visible in a lateral surface view such as that shown in Fig. 1A. AId is about $2 \mathrm{~mm}$ in length and extends slightly farther anteriorly than does AIv. Posteriorly, AId and AIv are replaced by AIp.

In horizontal section (Fig. 1B) the lamina dissecans is recognizable as a cell sparse zone between layers II/III and V in AIv and AId. Due to curvature of the brain surface in the region $\mathrm{AId}$ and $\mathrm{AIV}$, coronal sections such as those of Figs IC and ID are oriented obliquely rather than perpendicularly to the pial surface, and thus exaggerate the width of layers $\mathrm{V}$ and VI. As illustrated in Figs 1C and 1D, layers II and III of AIv are not distinct from one another and a prominent lamina dissecans separates layer II/III from layer $\mathrm{V}$ throughout AIv. In dorsally adjacent AId, layers II and III become clearly separated from each other and the lamina dissecans is gradually replaced by a granular layer IV which is prominent in the lateral precentral area dorsal to AId. These latter two features distinguish AId from AIv and, according to Sanides, ${ }^{54}$ are definitive characteristics of proisocortex versus periallocortex. Thus, AIv is a periallocortical region by virtue of its position adjacent to the allocortex primitivus of primary olfactory cortex, its poorly developed outer cell stratum (layers II/III) and the presence of a lamina dissecans. AId is a proisocortical region due to its position adjacent to lateral precentral isocortex, its relatively well-developed outer cell stratum (distinct layers II and III) and its incipient granular layer IV within the lamina dissecans. AId corresponds to area ail of Rose ${ }^{52}$ and Alv corresponds to his area ai2, as was previously noted by Krettek \& Price. ${ }^{35}$

The claustrum lies between layer VI and the external capsule throughout AId and AIv (see Fig. 1D) and becomes noticeably thinner in AIp. The endopiriform nucleus is a ventral portion of the claustrum which lies deep to the primary olfactory cortex. ${ }^{36}$

\section{Experimental results: dorsal agranular insular cortex}

Brains 80099 and 80065 represent HRP deposits into dorsal and ventral portions of AId, respectively.

Brain 80099. The deposit site in brain 80099 (Fig. $2 \mathrm{~A}$ ) is centered in the middle of AId and all layers are affected. There is some involvement of the dorsally adjacent lateral precentral isocortex.

In contralateral AId labelled pyramidal cells are found in layers III and V (Fig. 3A-F), continuously distributed between the anterior and posterior borders of AId but present in greatest number at the same level as the deposit site. A total of 88 labelled cells was found in eleven sections examined, $83 \%$ of which were seen in layer $\mathrm{V}$ (usually just deep to the lamina dissecans) and $17 \%$ in layer III. Labelled fibers were seen in the corpus callosum from the region of the deposit site across to contralateral AId, where they could be seen entering the gray matter.

In medial frontal cortex, labelled cells are seen bilaterally in layers III and $\mathrm{V}$ of the prelimbic and dorsal anterior cingulate areas (Fig. 3A-F). At all levels where cells are seen in the anterior cingulate region they are also present in the most medial part of the medial precentral isocortex, usually in layer $\mathrm{V}$.

In the posterior agranular insular cortex (AIp) labelled pyramidal neurons are present ipsilaterally in layers III, V and VI and are most numerous in its anterior portion (Fig. 3G-M). Anteriorly, labelled cells first appear in AIp just caudal to the level of the genu of the corpus callosum. This is the same level at which labelled cells are no longer seen in contralateral agranular insular cortex.

In the thalamus, labelled neurons are numerous in the posterior portion of the medial segment of the mediodorsal nucleus (MDmp) and are seen more sparsely in nuclei central medial, rhomboid, gelatinosus, ventromedial and parafascicularis (Fig. 3I-L). Labelled fibers in the inferior thalamic peduncle could be followed into thalamic nuclei containing labelled cells.

In the amygdala, labelled cells are abundant in the anterior division of the basolateral nucleus and are present as well in the lateral nucleus (Fig. 3H-L).

In the ventral caudal diencephalon labelled cells are found in the lateral hypothalamus and posterior hypothalamus (Fig. 3M-N).

In the brainstem the dorsal raphe nucleus contains labelled cells (Fig. 30).

Brain 80065 . The deposit site in experiment 80065 (Fig. 2B) is similar to that in 80099 but is situated more ventrally within Ald and is not quite as extensive rostrally. All layers of AId are affected throughout much of the extent of the deposit site. Posterior to its center, the HRP deposit site encroaches slightly on the lateral precentral isocortex dorsal to AId.

The distribution of labelled cells is similar to that in brain 80099 , with the addition of labelled cells in the parabrachial nucleus (Fig. 4). In contralateral AId and medial frontal cortex, labelled cells are most numerous at the same $a-p$ level as the deposit site. The commissural projection from contralateral AId was found to arise $87 \%$ from pyramidal cells in outer layer $\mathrm{V}$ and $13 \%$ from pyramidal cells in layer III (a total of 40 cells was found in six sections examined).

Further considerations. The results of other experiments in which the HRP deposit site was wholly or largely located in AId were substantially the same as those reported above for brains 80099 and 80065 . Analysis of all such brains warrants the conclusions that AId receives cortical input from contralateral AId, dorsal regions of medial frontal cortex (bilater- 
ally and topographically) and ipsilateral AIp. The major thalamic input is from MDmp, with sparse to moderate projections arising in the other nuclei mentioned above. Substantial amygdaloid projections originate in the lateral and anterior basolateral nuclei. The lateral and posterior hypothalamic areas, dorsal raphe nucleus, parabrachial nucleus and locus coeruleus were sometimes labelled in cases of HRP deposits into AId.

\section{Experimental results: rentral agranular insular cortex}

Brains 80066 and 80067 represent deposits into the most anterior and posterior portions of AIv, respectively.

Brain 80066. The deposit site in experiment 80066 (Fig. 2C) is restricted to Alv except for slight encroachment upon the orbital cortex and extends from its anterior boundary to near its posterior limit. Anteriorly, layers I-III are most extensively involved while at the center of the deposit site and posterior to it, layers I-V are involved. There is no encroachement upon primary olfactory cortex but the ventral portion of AIv which curves around the fundus of the rhinal fissure is affected.

In contralateral Alv labelled pyramidal neurons are seen in layers III and V, most abundantly at the same level as the deposit site (Fig. 5.B-D). In five sections examined, a total of 37 cells was found, $81 \%$ in layer $\mathrm{V}$ (usually just deep to the lamina dissecans) and $19 \%$ in layer III (the deep portion of layer II/III). Labelled fibers were seen in the corpus callosum, from the deposit site across to contralateral AIv.

In medial frontal cortex labelled cells are present bilaterally in the medial orbital and infralimbic areas, usually in layers II and III, and in layers III and $\mathrm{V}$ of the prelimbic area (Fig. 5,A-E). Cells in the medial orbital area are most numerous anterior to the deposit site.

Labelled pyramidal neurons are seen in layers III, $V$ and VI throughout the length of AIp (Fig. 5.F-L). evenly distributed from a level just posterior to the

Fig. 1. Location and structure of agranular insular cortex in the hamster. Part $\mathrm{A}$ is a lateral surface view of the right side of the brain, indicating cytoarchitectonic boundaries as well as the planes of sections shown in $\mathrm{B}, \mathrm{C}$ and $\mathrm{D}$.

\section{Abbreviations used on Figures}

$\begin{array}{ll}\text { ac } & \text { anterior commissure } \\ \text { ACd } & \text { dorsal portion. anterior cingulate cortex } \\ \text { ACV } & \text { ventral portion, anterior cingulate cortex } \\ \text { AD } & \text { anterodorsal thalamic nucleus } \\ \text { AHA } & \text { amygdalo-hippocampal area } \\ \text { AId } & \text { dorsal agranular insular cortex } \\ \text { AIp } & \text { posterior agranular insular cortex } \\ \text { AIv } & \text { ventral agranular insular cortex } \\ \text { AM } & \text { anteromedial thalamic nucleus } \\ \text { AV } & \text { anteroventral thalamic nucleus } \\ \text { bc } & \text { brachium conjunctivum } \\ \text { BLa } & \text { anterior portion, basolateral amygdaloid nucleus } \\ \text { BLp } & \text { posterior portion, basolatcral amygdaloid nucleus } \\ \text { BM } & \text { basomedial amygdaloid nucleus } \\ \text { CE } & \text { central amygdaloid nucleus } \\ \text { CeL } & \text { central lateral thalamic nucleus } \\ \text { CL } & \text { claustrum } \\ \text { CM } & \text { central medial thalamic nucleus } \\ \text { cp } & \text { cerebral peduncle } \\ \text { cst } & \text { corticospinal tract } \\ \text { C1 } & \text { anterior cortical amygdaloid nucleus } \\ \text { C2 } & \text { posterolateral cortical anygdaloid nucleus } \\ \text { C3 } & \text { posteromedial cortical amygdaloid nucleus } \\ \text { DP } & \text { dorsal peduncular cortex } \\ \text { DR } & \text { dorsal raphe nucleus } \\ \text { E } & \text { endopiriform nucleus } \\ \text { ERC } & \text { entorhinal cortex } \\ \text { f } & \text { fornix } \\ \text { fm } & \text { forceps minor of corpus callosum } \\ \text { G } & \text { gelatinosus (or submedial) thalamic nucleus } \\ \text { HL } & \text { lateral hypothalamus } \\ \text { IL } & \text { infralimbic cortex } \\ \text { L } & \text { lateral amygdaloid nucleus } \\ & \end{array}$

LH lateral habenular nucleus

M medial amygdaloid nucleus

MDma anterior portion, medial segment of mediodorsal thalamic nucleus

MDmp posterior portion, medial segment of mediodorsal thalamic nucleus

MH medial habenular nucleus

$\mathrm{ml}$ medial lemniscus

MO medial orbital cortex

$\mathrm{mt}$ mammillothalamic tract

$\mathrm{PaC}$ paracentral thalamic nucleus

PB1 lateral portion, parabrachial nucleus

PBm medial portion, parabrachial nucleus

PC medial precentral cortex

PF parafascicular thalamic nucleus

PL prelimbic cortex

POCp posterior primary olfactory cortex

PR perirhinal cortex

PT parataenial thalamic nucleus

$\mathrm{PVa}$ anterior portion, paraventricular thalamic nucleus

$\mathrm{PVp}$ posterior portion, paraventricular thalamic nucleus

RE reuniens thalamic nucleus

RH rhomboid thalamic nucleus

sd supramammillary decussation

sm stria medullaris

TR entorhinal cortical area TR

TT taenia tecta

VCD ventral caudal diencephalon

VM ventromedial thalamic nucleus

28L lateral entorhinal cortical area $28 \mathrm{~L}$

$28 \mathrm{~L}^{\prime}$ lateral entorhinal cortical area $28 \mathrm{~L}^{\prime}$ 

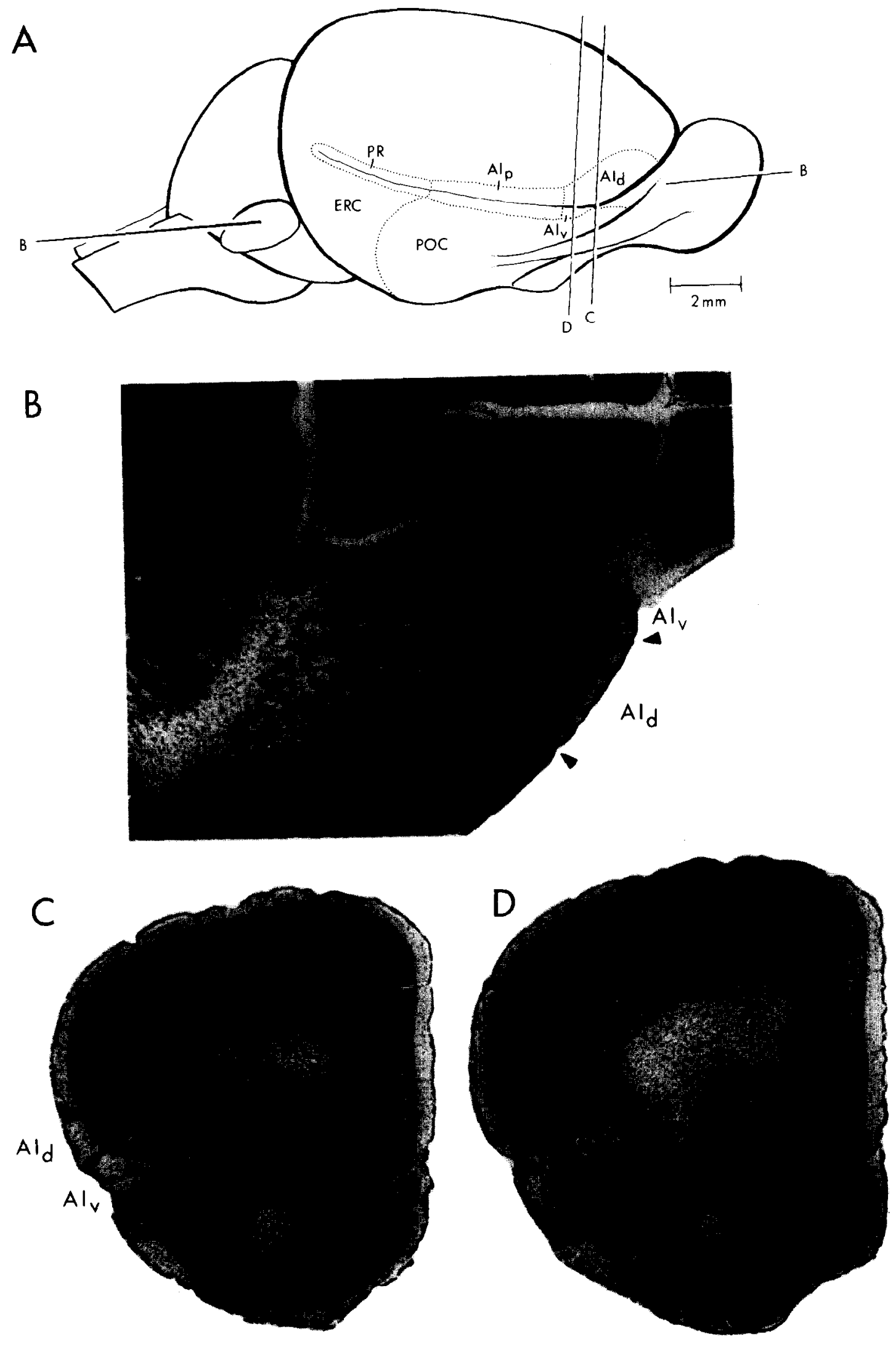

Fig. 1. 

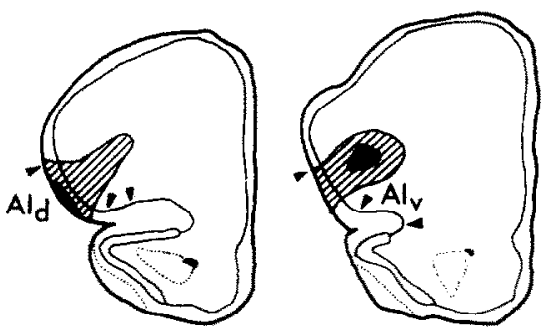

A 80099
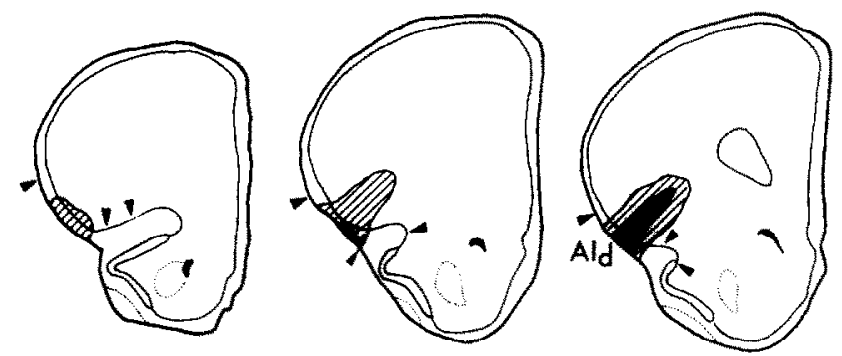

B 80065
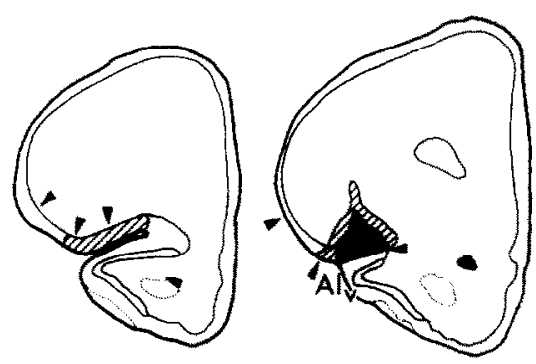

C 80066
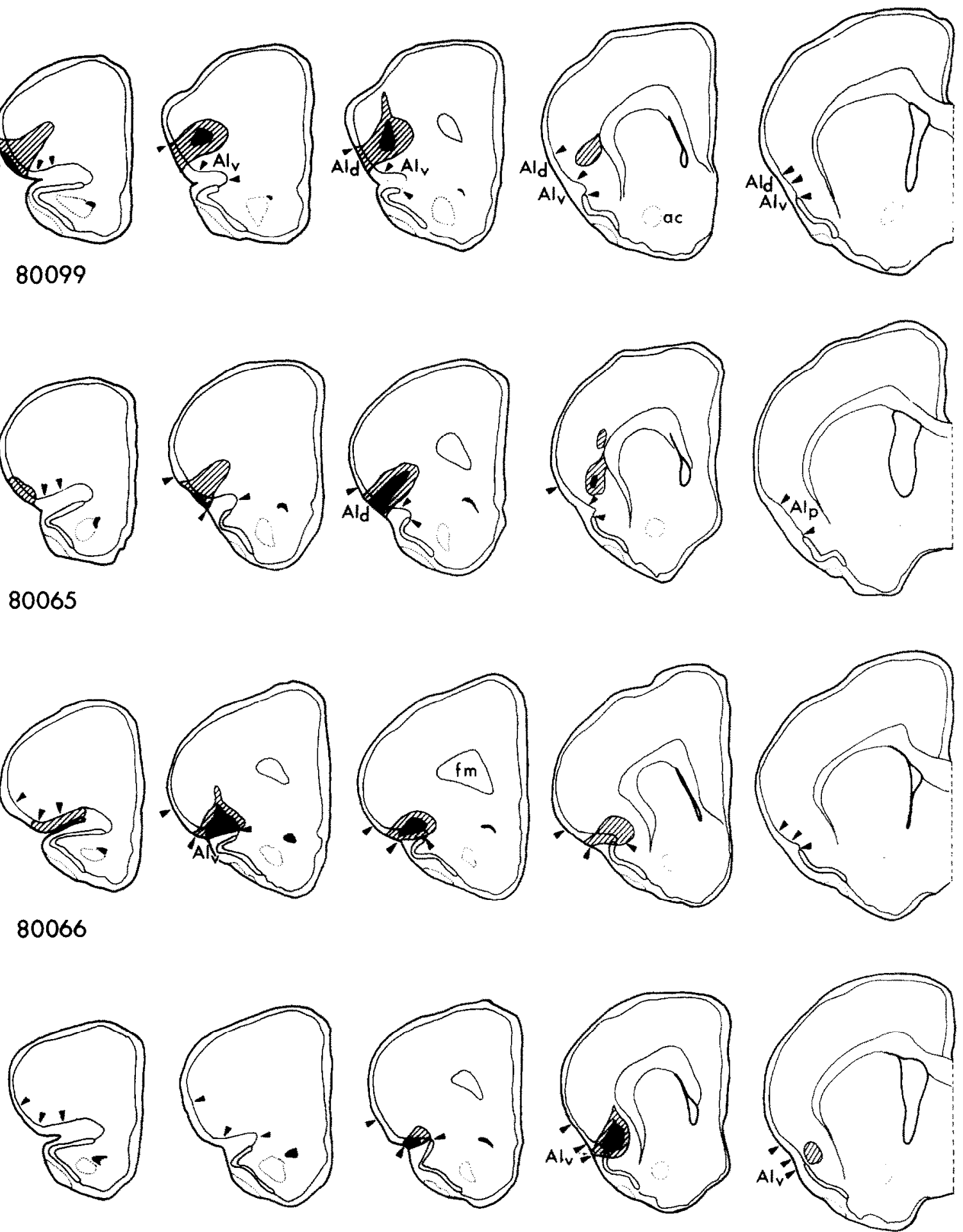

D 80067

Fig. 2. Representative coronal sections illustrating the horseradish peroxidase deposit site in each of the four brains described in the text. Here, and in Figs 3-6, solid-shaded areas represent zone $I$ of the deposit site (see text) while stripes represent zone 2. 
genu of the corpus callosum to the perirhinal cortex. A few cells were found in the underlying claustrum as well. Pyramidal neurons are seen in layers III and V of perirhinal cortex (Fig. $5 \mathrm{M}-\mathrm{O}$ ).

Tertiary olfactory areas (those receiving direct projections from the olfactory bulb) containing labelled neurons include the posterior primary olfactory cortex (terminology of Heimer $^{22}$ ) (Fig. 5F-K), area $28 \mathrm{~L}$ (terminology of $\mathrm{Haug}^{20}$ ) of lateral entorhinal cortex (Fig. 5M-O), and the posterolateral cortical amygdaloid nucleus, C2 (terminology of Scalia \& Winans ${ }^{57}$ ) (Fig. 5J-K). In posterior primary olfactory cortex, 55 cells were found in 14 sections, $71 \%$ in layer III, $29 \%$ in layer IIb. The endopiriform nucleus deep to posterior primary olfactory cortex contains a few labelled cells (Fig. 5,H-I).

Brain 80067 . In experiment 80067 , the deposit site is centered in the posterior portion of AIv and involves layers I-VI (Fig. 2D). There is no encroachment upon the anterior primary olfactory cortex ventrally.

The distribution of labelled cells was similar to that in brain 80066 with the following qualifications.

In contralateral AIv, labelled pyramidal cells are most numerous at about the same level as the deposit site and are not seen as far rostrally as in experiment 80066 (Fig. 6C-D). A total of 18 cells was found in six sections examined, $100 \%$ of which were in layer $\mathrm{V}$.

In medial frontal cortex, layers II and III of the medial orbital area contain cells bilaterally (Fig. $6 \mathrm{~A}-\mathrm{C})$ and these are located more posteriorly than those of experiment 80066 .

Among the tertiary olfactory areas, posterior primary olfactory cortex contains labelled cells in layers IIb and III from the level of the genu of the corpus callosum posteriorly to its border with entorhinal cortex (Fig. $6 \mathrm{E}-\mathrm{K}$ ). 'This is in contrast to experiment 80066 , in which labelled cells are very sparse in caudal portions of posterior primary olfactory cortex. In brain 80067 a total of 59 labelled cells was found in ten sections examined, $66 \%$ in layer III and $34 \%$ in layer IIb. The lateral entorhinal cortex contains labelled cells in areas $28 \mathrm{~L}$ and $28 \mathrm{~L}^{\prime}$ (Fig. $6 \mathrm{~L}-\mathrm{M}$ ). The posterolateral cortical nucleus of the amygdala, $\mathrm{C} 2$, and the endopiriform nucleus also contain labelled cells (Fig. 6F-K).

Further considerations. Other experiments in which the HRP deposit was wholly or largely confined to AIv gave results which were similar to those reported above for brains 80066 and 80067 . Analysis of all such brains warrants the conclusions that AIv receives cortical input from contralateral AIv, ventral portions of medial frontal cortex (bilaterally and topographically organized; deposits involving the most ventral portion of $\mathrm{AIV}$, which curves around the fundus of the rhinal fissure, resulted in the greatest number of labelled cells in infralimbic cortex), ipsilateral AIp and perirhinal cortex. The major thalamic input is from MDma, with other sparser projections originating in the nuclei mentioned above. Tertiary olfactory inputs arise from the posterior primary olfactory cor- tex, endopiriform nucleus, lateral entorhinal cortex and posterolateral cortical amygdaloid nucleus. All brains had labelled cells in the dorsal raphe nucleus, and some had cells in locus coeruleus.

\section{Control brains}

In our experiments an angled dorsal approach was most often used to direct the HRP-filled micropipette into either AId or AIv. Often there was some labelling along the pipette track in areas dorsal to AId. In order to assess the effect of such track labelling on our results, deposits were made into these dorsallylocated areas by lowering the pipette along the same trajectory as usual but not as far ventrally. In two such cases (not illustrated), the deposit site was situated just dorsal to the posterior portion of AId at a level midway between the head of the caudate nucleus and the genu of the corpus callosum. Extrapolating from studies in rats, this corresponds to the granular insular taste area and sensory-motor isocortex. In brain 80021 deep cortical layers were affected and in brain 80100 more superficial layers were involved.

In the cortex, labelled cells were seen in ipsilateral dorsal cortical regions adjacent to the deposit site and contralaterally in the same region as the deposit site. In the thalamus, labelled neurons were most numerous in the basal (taste) portion of the ventromedial nucleus, and in the paracentral, ventral lateral and ventrobasal nuclei. No other labelled cells were seen in any other areas in either of these brains.

HRP deposits in anterior portions of AIp produced no labelling in the mediodorsal thalamic nucleus, confirming Krettek \& Price's ${ }^{35}$ autoradiographic findings in the rat.

\section{DISCUSSION}

The present study has reported previously undocumented inputs to AId from the anterior cingulate and medial precentral areas bilaterally, the posterior agranular insular area ipsilaterally, the lateral nucleus of the amygdala, posterior hypothalamus, and medial and lateral parabrachial nuclei; to AIv from the medial orbital and infralimibic areas bilaterally, and lateral entorhinal cortex ipsilaterally.

The most striking findings of this study are that AId and AIv each receive a distinct combination of inputs, and that inputs to AId and AIv from a common source usually arise from completely or partially separated regions of that source.

\section{Cytoarchitectonics}

Brodmann and Vogt introduced the distinction between isogenetic (or homogenetic) and allogenetic (or heterogenetic) cortical areas. As Stephan \& Andy ${ }^{61}$ point out, "the word 'allocortex' (the other cortex) functioned from the beginning as a collective term specifying the entire region of the 'nonisocortex'... therefore, the allocortex has become more and more subdivided." In contemporary usage the term 

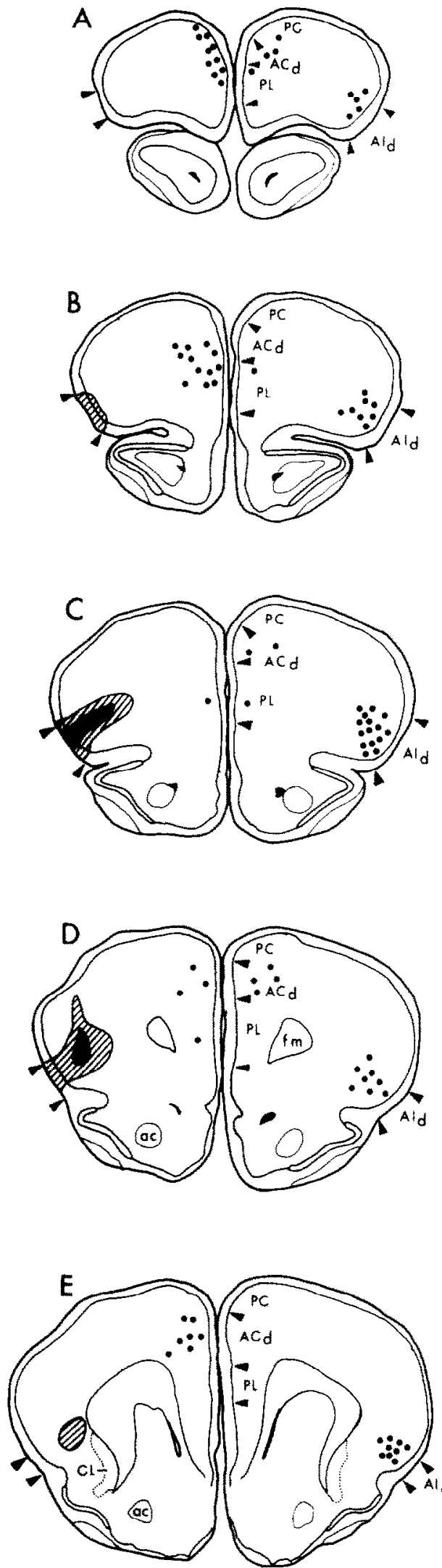
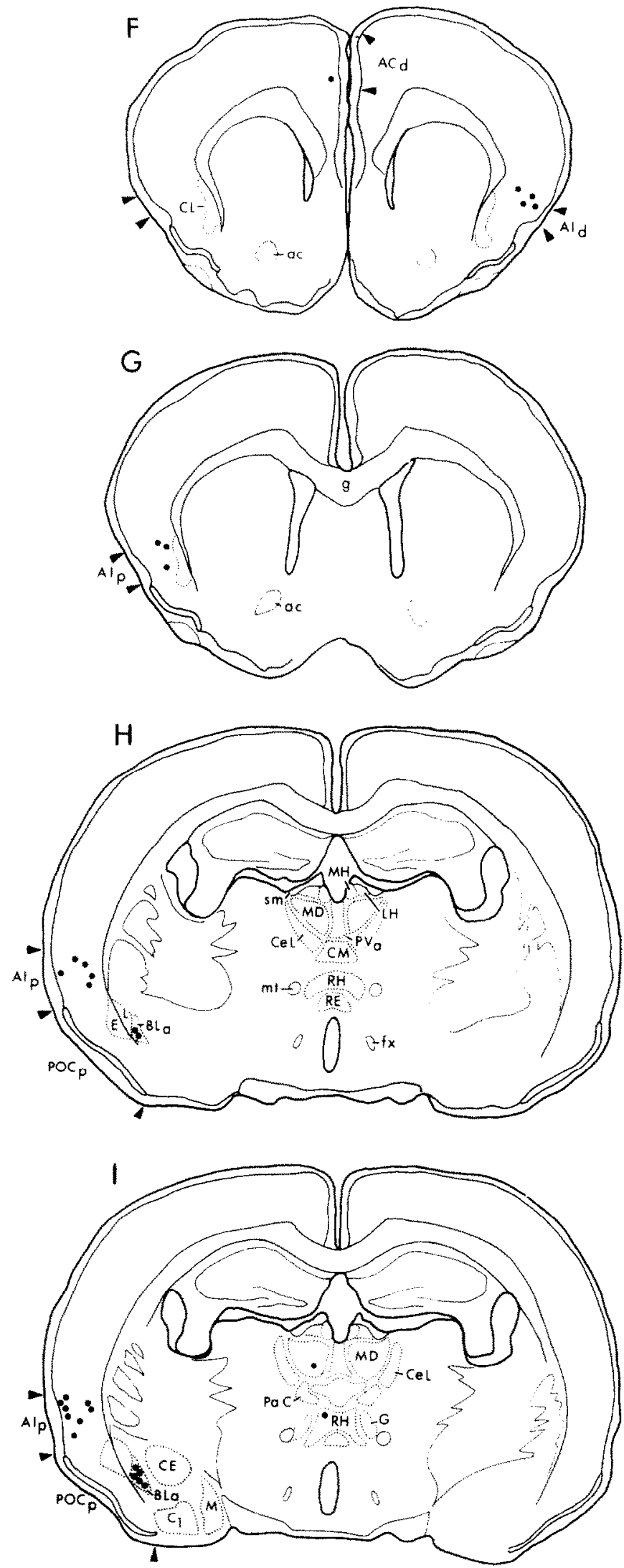

Fig. 3. 

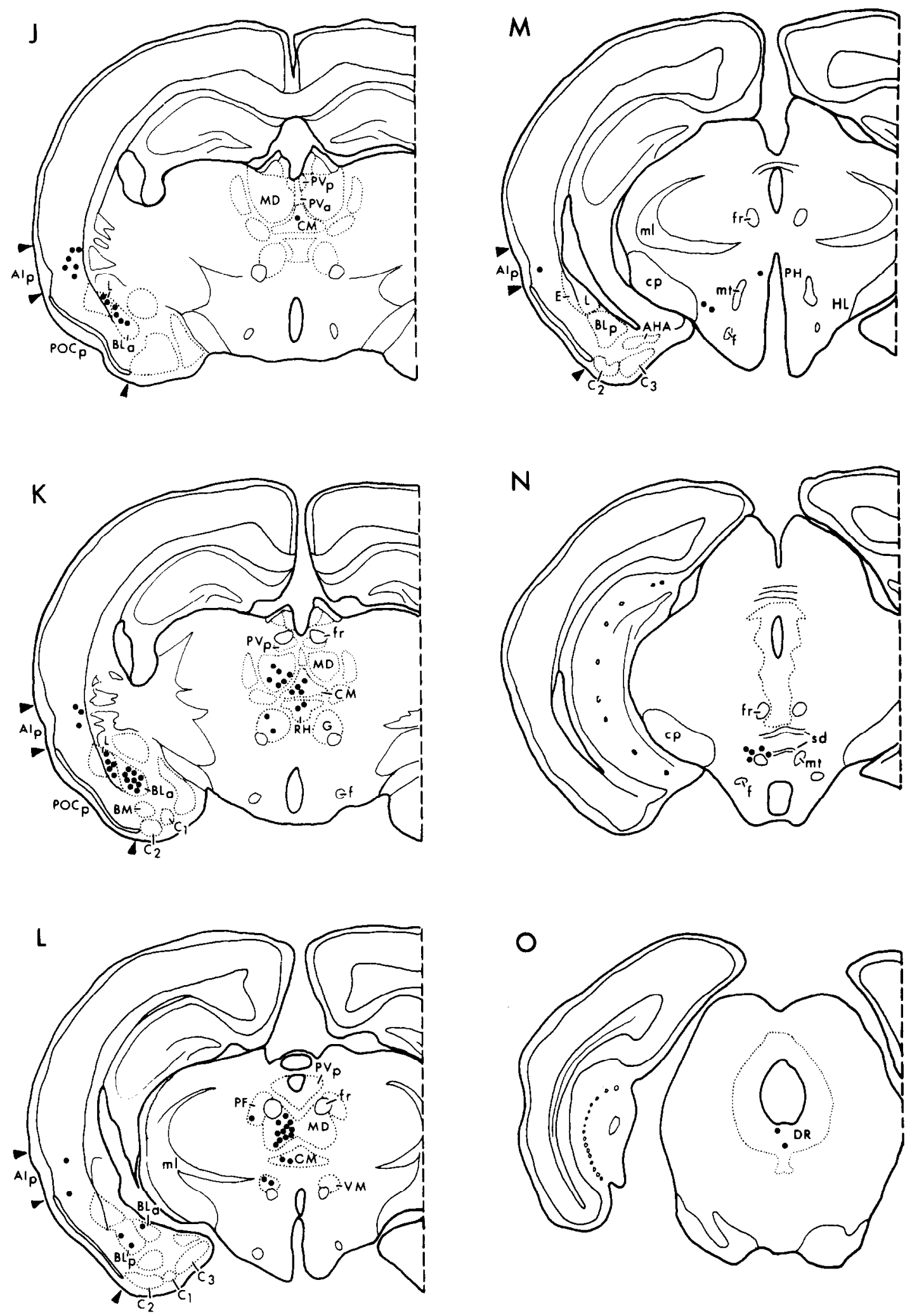

Fig. 3. Brain 80099 . A series of representative coronal sections illustrating the distribution of horseradish peroxidase labelled neurons. 
A
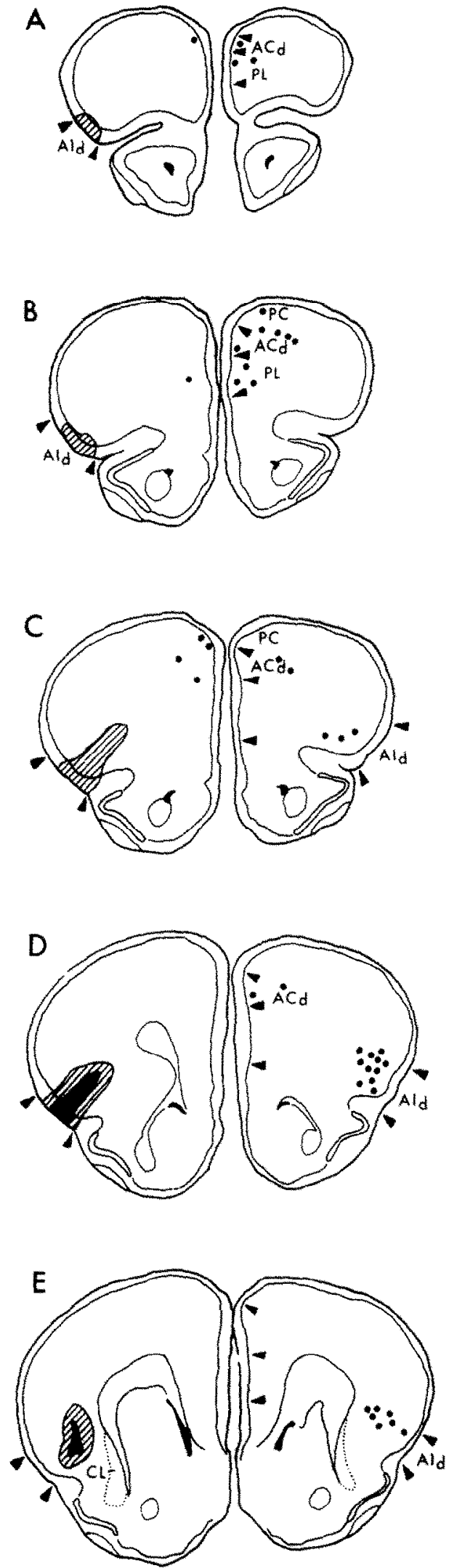

F

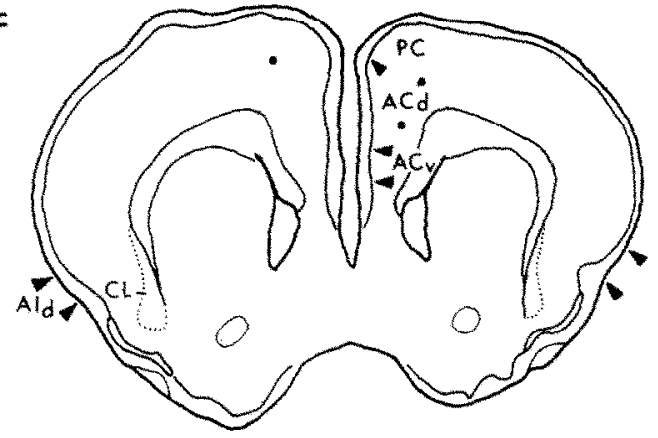

G
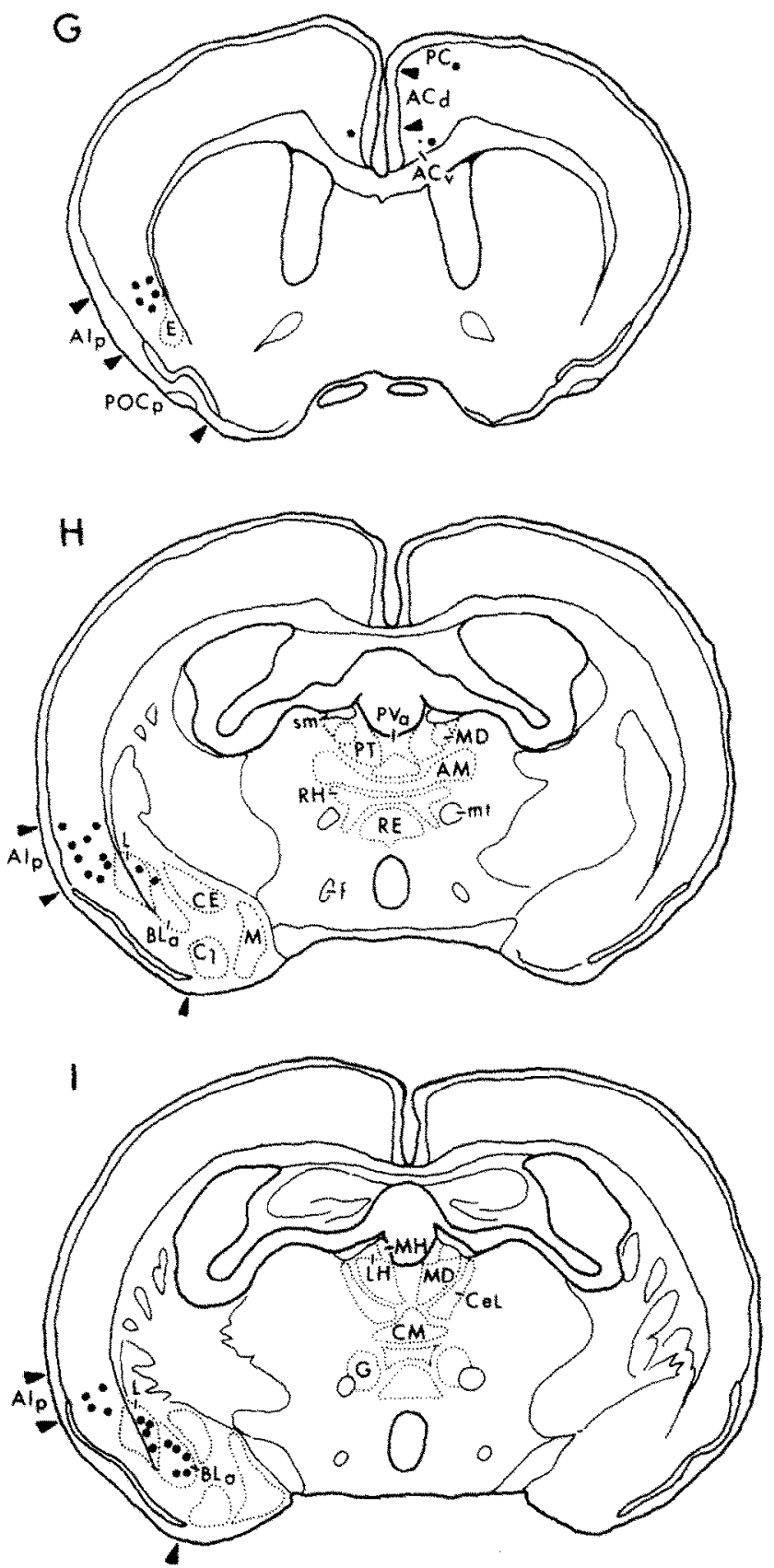

Fig. 4. 

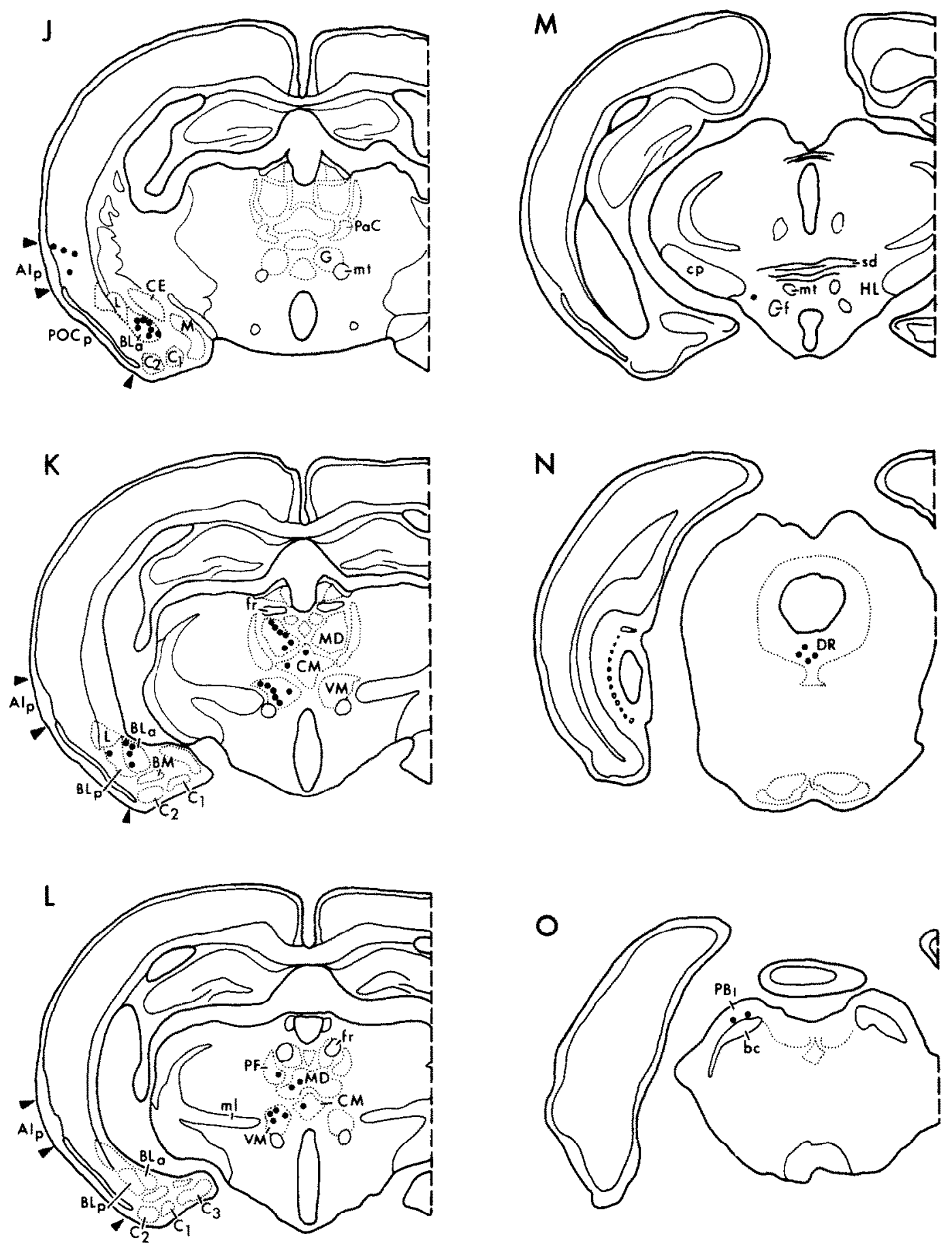

Fig. 4. Brain 80065 . A series of representative coronal sections illustrating the distribution of horseradish peroxidase labelled neurons.

allocortex commonly refers only to paleocortex and archicortex, or allocortex primitivus in the terminology of Stephan \& Andy. ${ }^{61}$ However, dorsally adjacent to these two zones lie cortical areas which are transitional in location and structure between allocortex primitivus and isocortex. These areas have been designated periallocortex and proisocortex. ${ }^{54}$
Frontally, there is a dorsalward progression from allocortex primitivus through periallocortex and proisocortex, laterally (from olfactory cortex through AIv and AId to lateral precentral isocortex) and medially (from the anterior hippocampus, or tacnia tecta, through infralimbic and anterior cingulate cortex to medial precentral isocortex). 
A

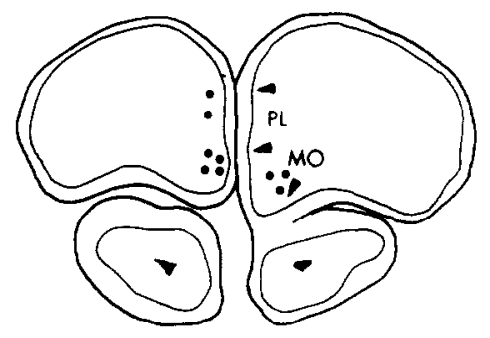

B

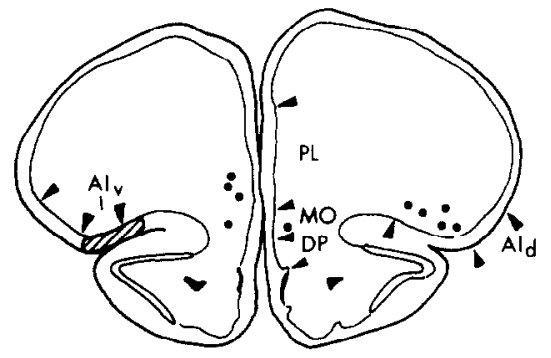

C

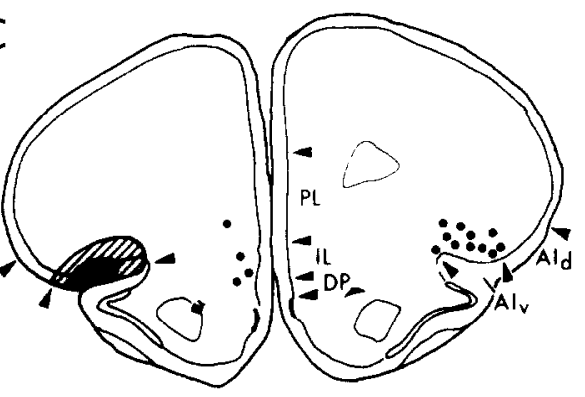

D

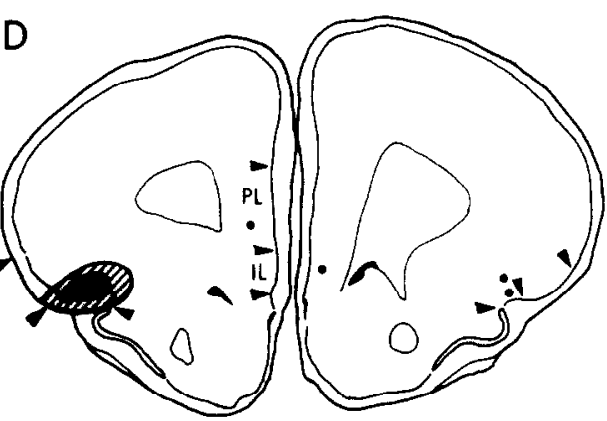

E

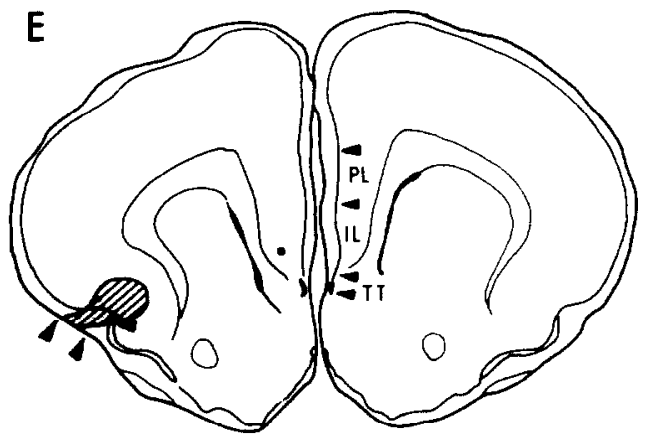

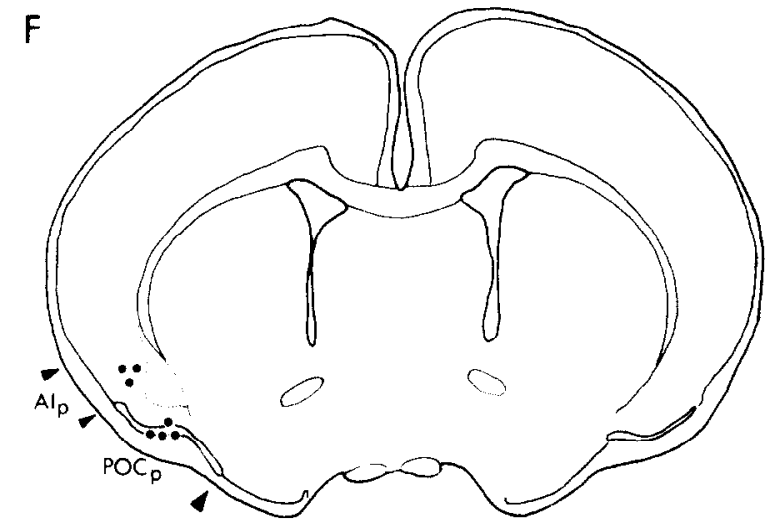
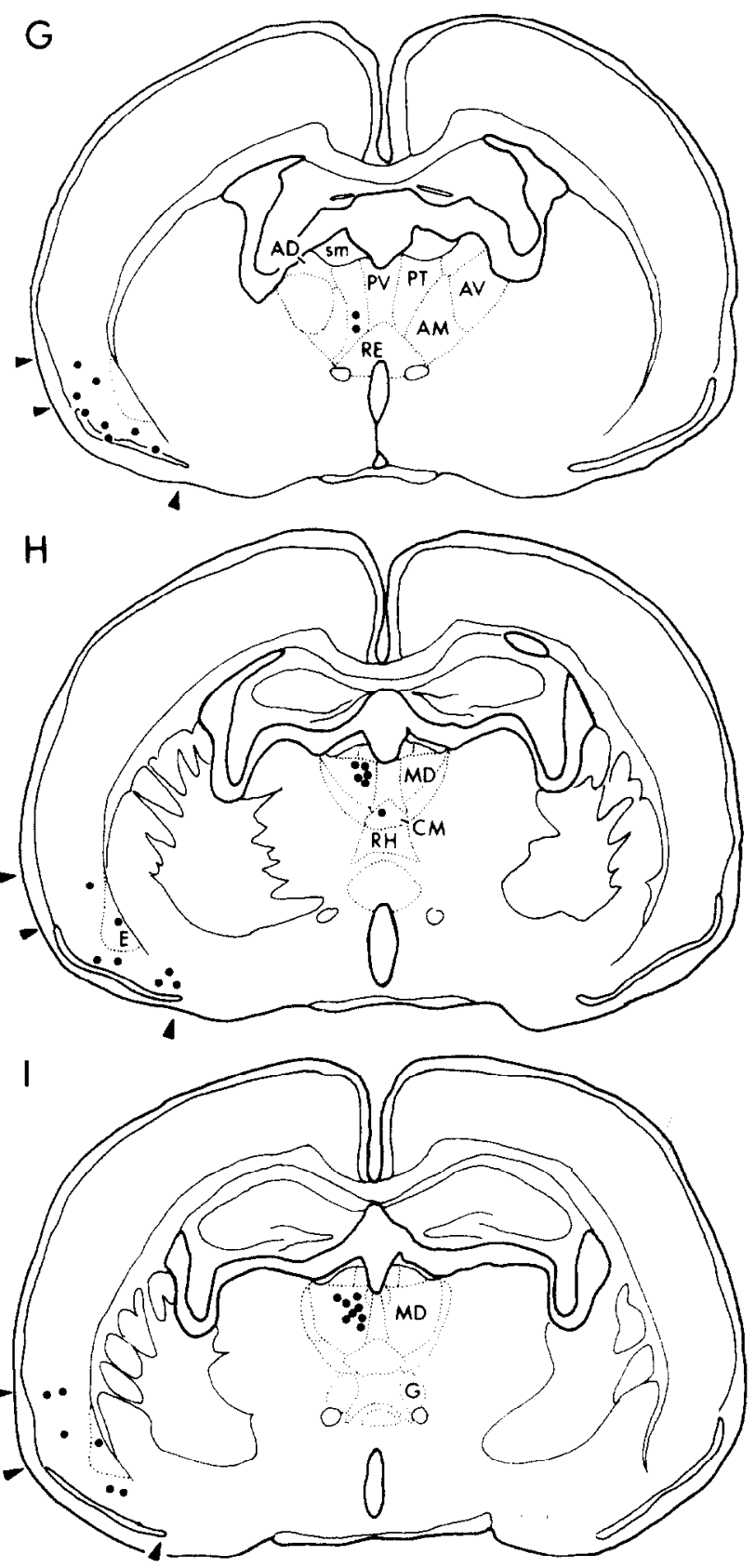

Fig. 5 . 

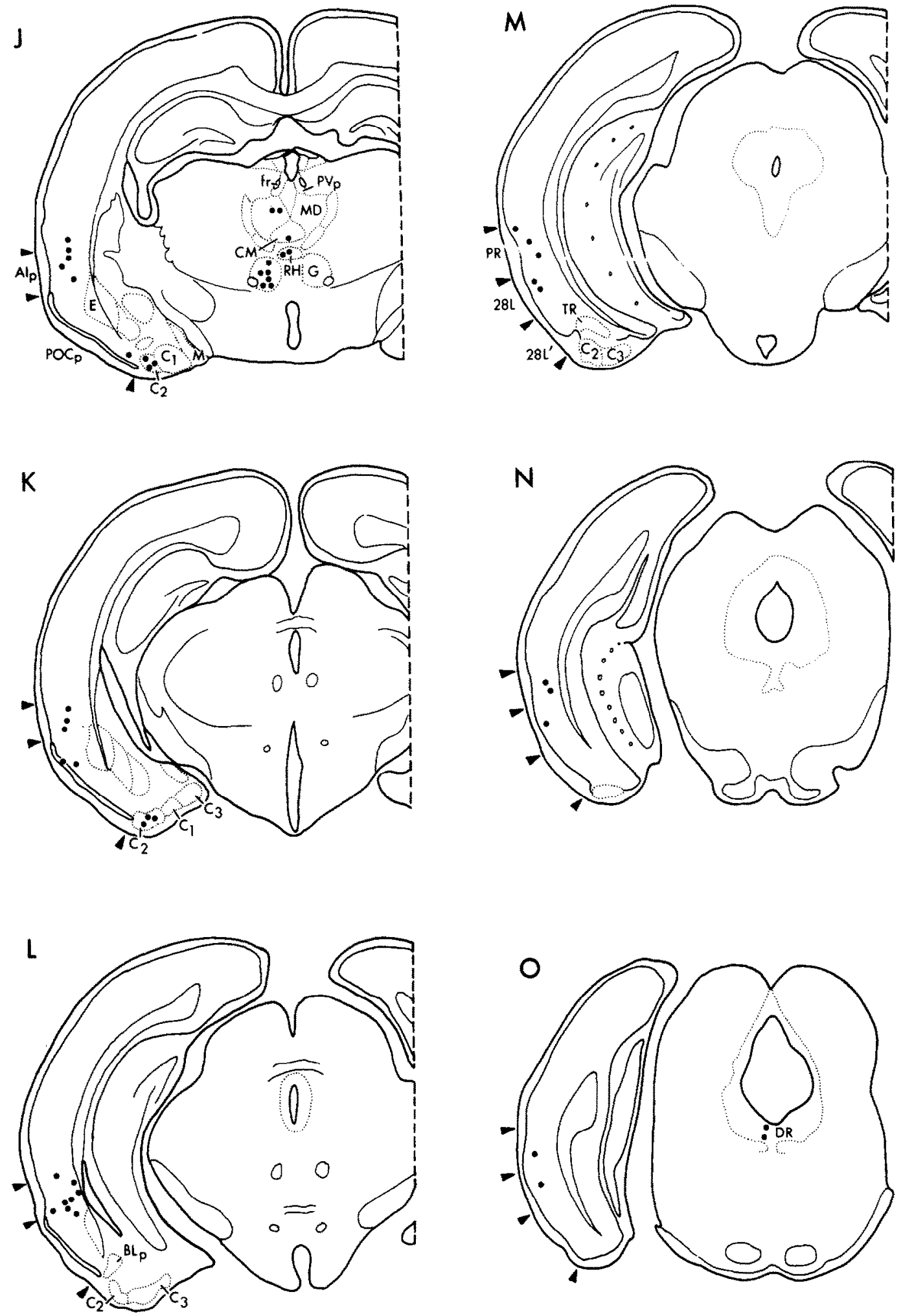

Fig. 5. Brain 80066. A series of representative coronal sections illustrating the distribution of horseradish peroxidase labelled neurons. 
The anterior agranular insular cortex (AId and AIv) is one portion of a continuous belt of agranular cortex that includes other periallocortical and proisocortical transition regions, as well as precentral motor isocortex. ${ }^{28}$ The transition areas are AIv and AId laterally, orbital cortex frontally, and infralimbic, prelimbic and anterior cingulate cortex medially. The precentral motor isocortex occupies the dorsolateral surface, extending from the anterior cingulate area to AId and thereby completing the belt.
Because they receive direct input from the mediodorsal thalamic nucleus, AId and AIv are usually considered to be part of the 'prefrontal' cortex, which was defined by Rose \& Woolsey ${ }^{53}$ as the total cortical projection area of MD. Although there is evidence that the rodent agranular insular cortex has functional properties similar to those of primate prefrontal cortex, ${ }^{41}$ the latter is characterized anatomically by its pronounced granularization whereas rodents have no such granular prefrontal cortex ${ }^{35}$ Based on
A

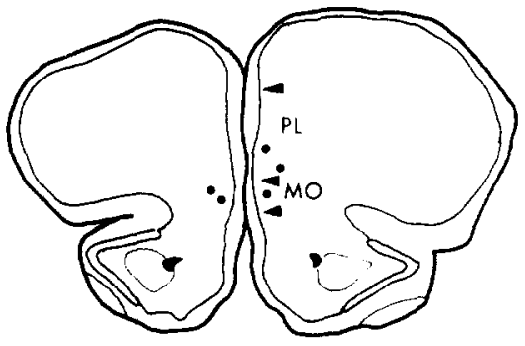

B

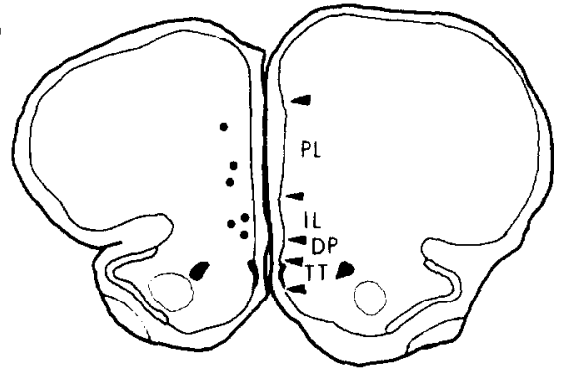

C
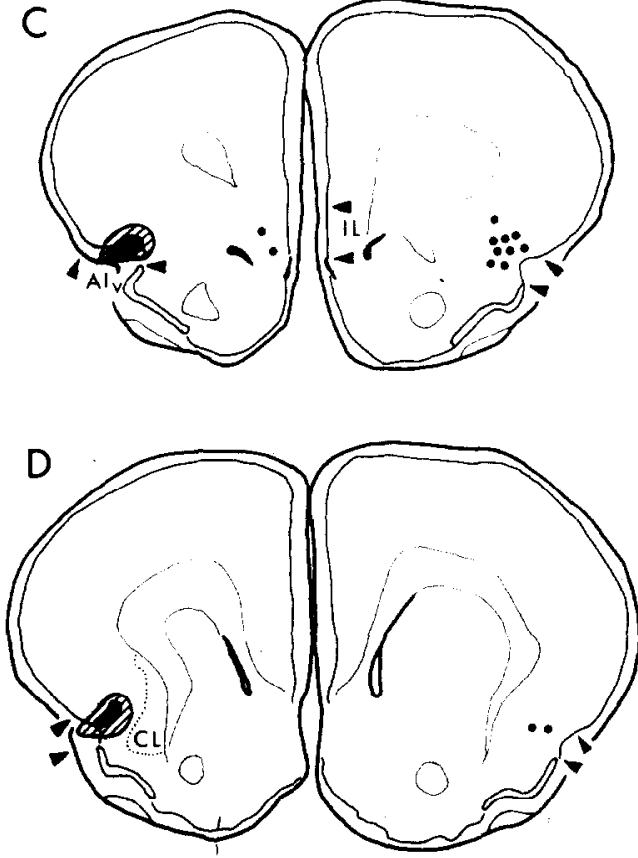

$E$
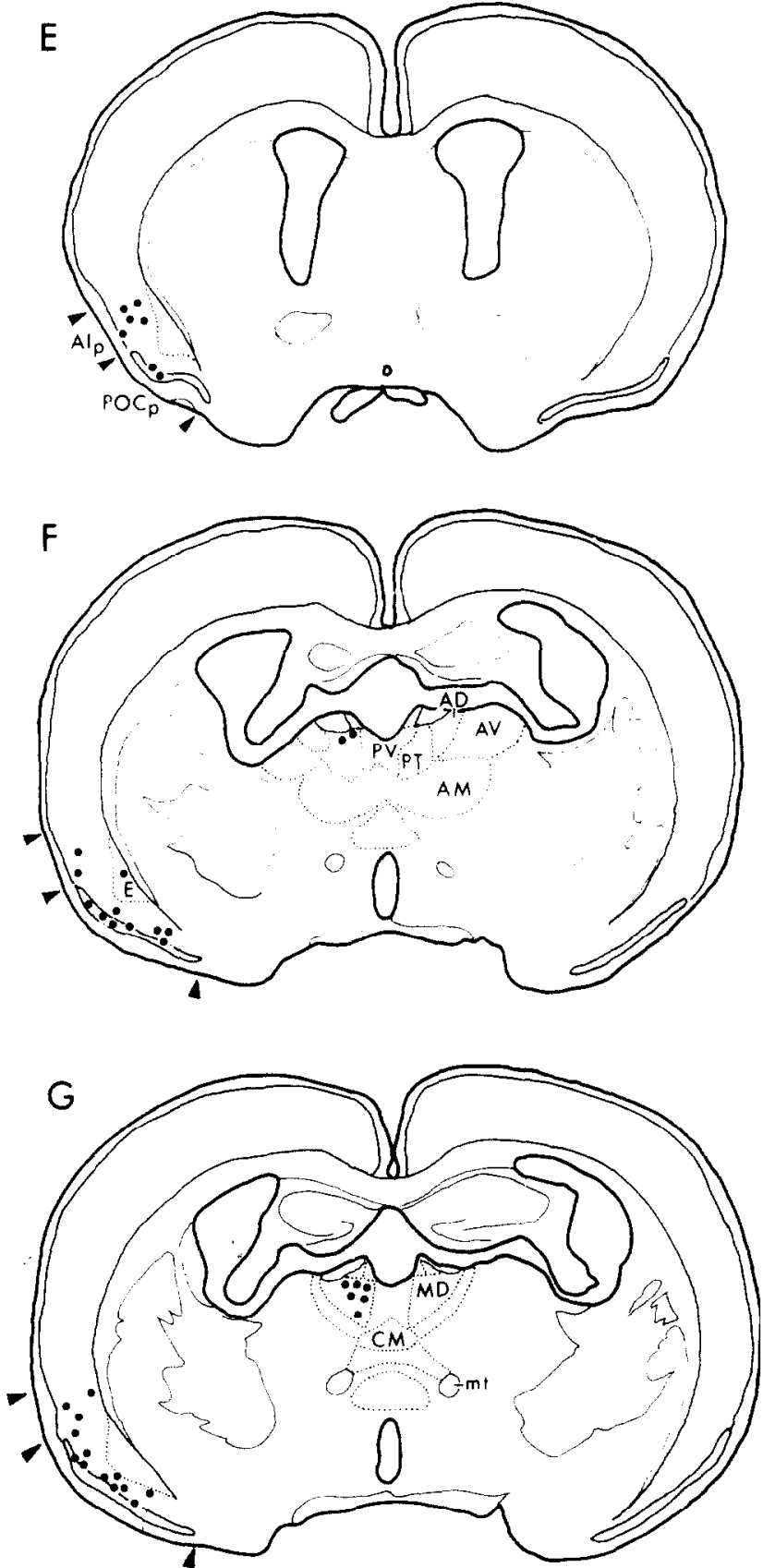

Fig. 6 . 

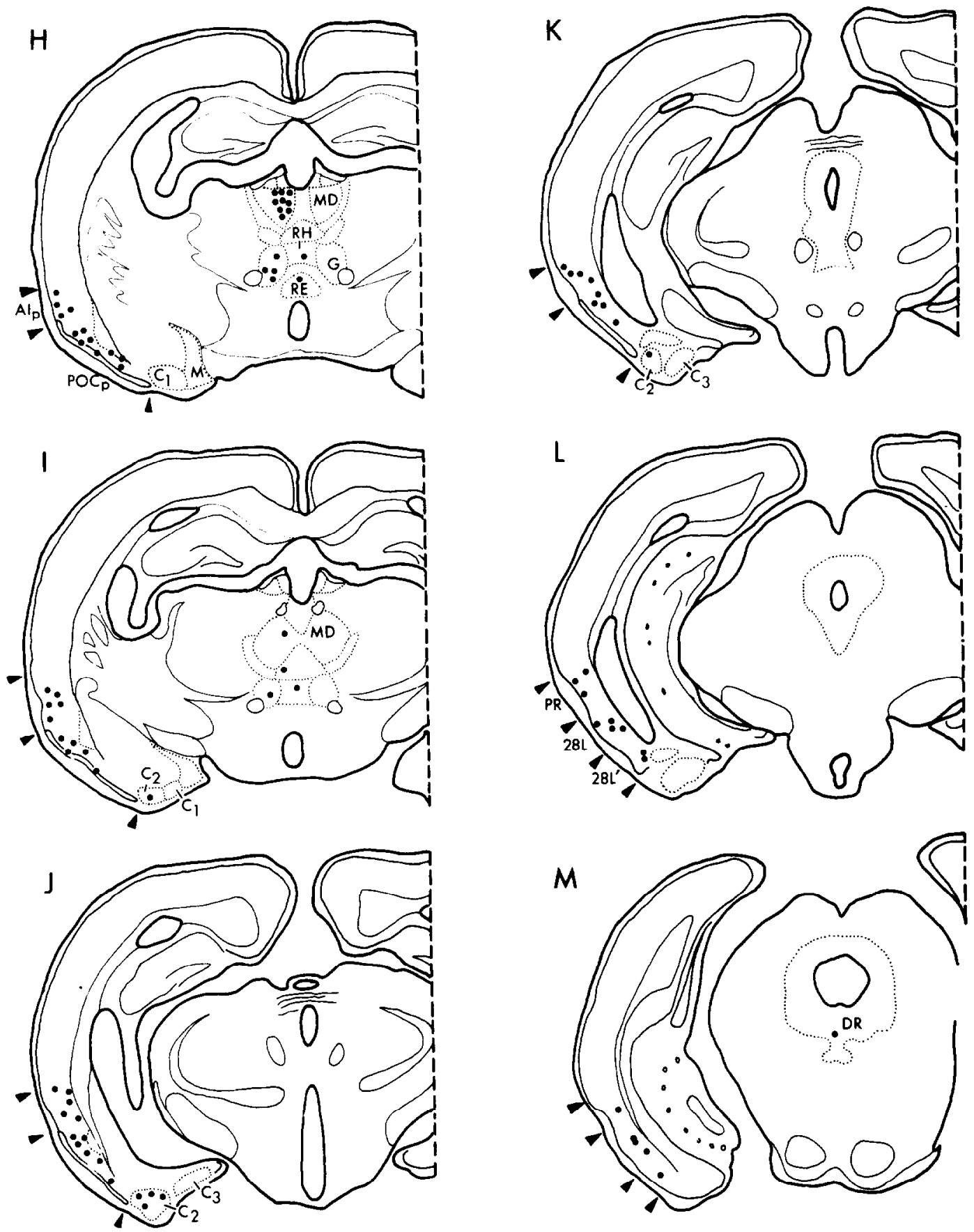

Fig. 6. Brain 80067 . A series of representative coronal sections illustrating the distribution of horseradish peroxidase labelled neurons.

comparative cytoarchitectonic studies, Sanides ${ }^{54}$ has suggested that granular prefrontal cortex originated from agranular prefrontal cortex. Therefore, it may be that in rodents definitive funtional properties of primate granular prefrontal cortex are present but are localized in presumptive granular zones within the agranular belt composed of insular, orbital and medial frontal cortex.

\section{Cortico-cortical connections}

Commissural input. Two previous reports exist concerning the commissural connections between ipsilateral and contralateral AId and AIv. The HRP study of Gerfen \& Clavier $^{17}$ showed that AId and AIv each receive input from contralateral agranular insular cortex but did not discuss the precise location (i.e. AId versus AIv), laminar distribution, or cell types of 
origin for either of these projections. Similarly, the autoradiographic study of Beckstead ${ }^{4}$ demonstrated commissural connections between ipsilateral and contralateral agranular insular cortex but the amino acid deposit sites (see his Figs 6 and 7) apparently included both AId and AIv.

Our results indicate that AId and AIv each receive input from their contralateral homotopical counterpart, with little or no overlap. Thus, for example, Ald receives input from contralateral AId but not from contralateral AIv. Furthermore, we found that the cells of origin for these projections are pyramidal neurons whose perikarya are located predominantly in outer layer V $\left(90^{\circ}\right)$ and rarely in layer III $\left(10^{\circ} \%\right.$. In
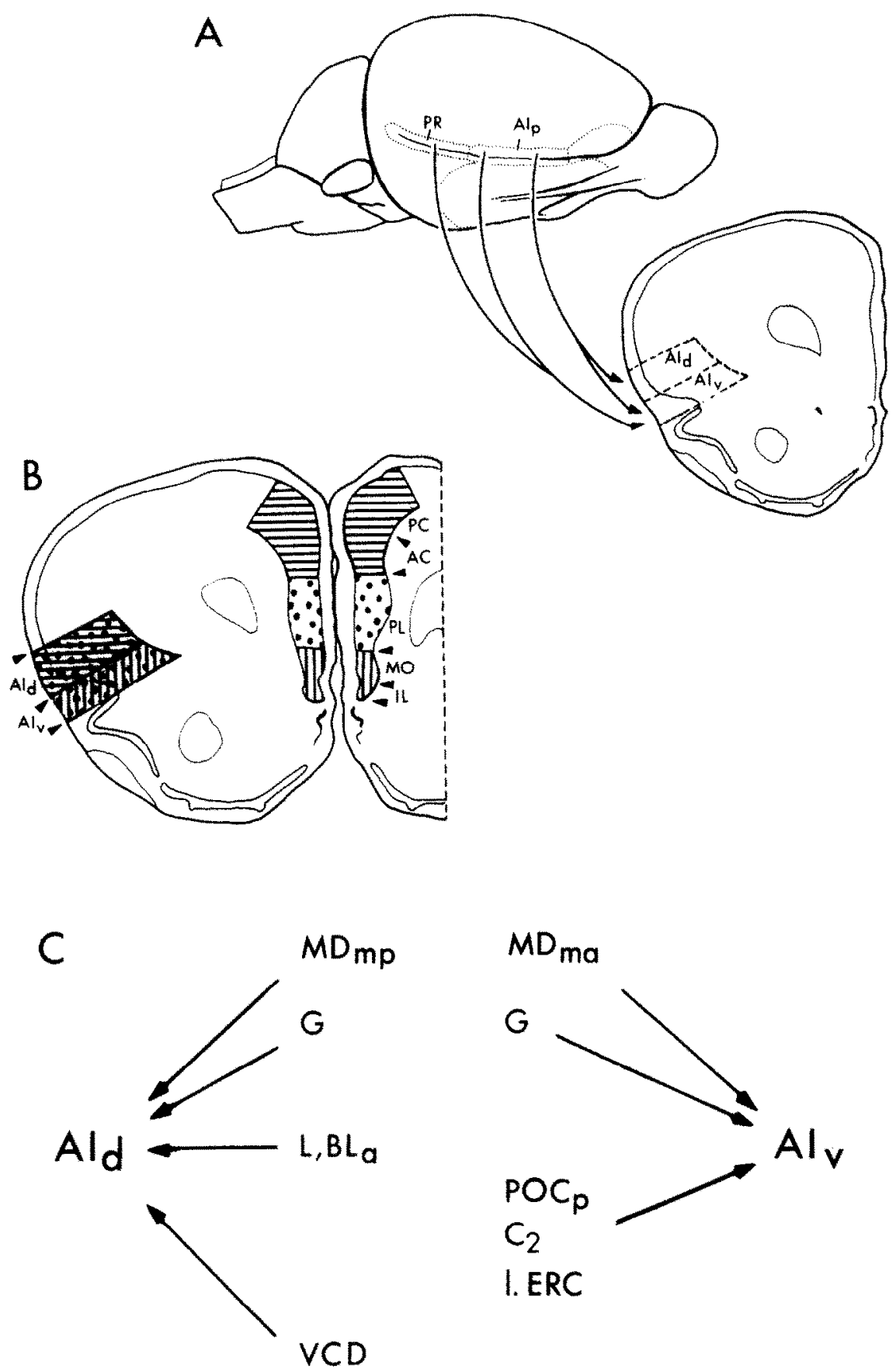

I. ERC

Fig. 7. Schematic summary of the major afferent connections of dorsal and ventral agranular insular cortex. In $A$ and $B$ are illustrated, respectively, the topographic organization of inputs from lateral and medial frontal transition cortical areas to AId and AIv. In C the other major inputs to Ald and AIv are diagrammed. 
contrast, Wise \& Jones ${ }^{66}$ found that the commissural projection of somatic sensory cortex in the rat originates from comparable numbers of layer III and layer $\mathrm{V}$ pyramidal cells, or possibly to a greater cxtent from layer III cells (see their Fig. 17). Differences in migration rate which are known to exist between isocortex and agranular insular cortex during neurogenesis ${ }^{24}$ could conceivably result in different laminar distributions of pyramidal neurons in these cortical areas, leading in turn to different laminar origins for the commissural projections of isocortex versus agranular insular cortex.

Medial frontal cortex. Projections from medial frontal cortical areas to AId and AIv have not been systematically investigated previously, but were partially treated in the autoradiographic study of Beckstead. ${ }^{4}$ $\mathrm{He}$ found a projection from the prelimbic area to layers I, III and VI of agranular insular cortex bilaterally, apparently to both AId and AIv (see his Fig. 2A-C). We have confirmed Beckstead's finding that AId and AIv each receive input from the prelimbic area bilaterally, and have further demonstrated bilateral inputs to AId from the anterior cingulate and medial precental areas dorsal to the prelimbic area, and to AIv from the infralimbic and medial orbital areas ventral to the prelimbic area. Thus, dorsallylocated areas of medial frontal cortex project to the dorsal agranular insular area while ventrally-located areas of medial frontal cortex project to the ventral agranular insular area, as shown schematically in Fig. 7 B.

Labelled neurons were most numerous in infralimbic cortex when the deposit site involved layers II and III of the most ventral portion of AIv, which curves around the fundus of the rhinal fissure and is adjacent to anterior primary olfactory cortex. In single sections, HRP-filled axons could be followed medially from superficial layers of AIv directly to the infralimbic area. Since some of our HRP deposits into AIv also encroached upon the anterior primary olfactory cortex to varying degrees, it is reasonable to question whether this area also receives input from infralimbic cortex. In our material, labelled fibers were never traced from anterior primary olfactory cortex to infralimbic cortex, but were instead directed to the ventral taenia tecta and dorsal peduncular cortex (terminology of Haberly \& Price $^{18}$ ) just ventral to infralimbic cortex. This is in accord with the findings of Haberly \& Price, ${ }^{19}$ in which injections of HRP into anterior primary olfactory cortex resultcd in labelled cells in these two areas but not in the infralimbic cortex. It must be noted, however, that their injections (see, for example, their Fig. 19) may not have significantly involved the most dorsal portion of anterior primary olfactory cortex, which is adjacent to AIv. Therefore, we cannot exclude a projection from infralimbic cortex to this area of primary olfactory cortex in addition to AIv.

Interestingly, the interconnected ventral areas AIv and infralimbic cortex both receive input from ter- tiary olfactory areas: primary olfactory cortex, entorhinal cortex and posterolateral cortical amygdala (our results; Haberly \& Price; $;^{18}$ Kevetter \& Winans; ${ }^{30}$ Krettek \& Price $^{36}$ ). No readily apparent parallel can be drawn regarding common inputs to the dorsal areas AId and anterior cingulate/medial precentral cortex.

Posterior agranular insular cortex and perirhinal cortex. Input to AIv from posterior agranular insular cortex (AIp) and perirhinal cortex (PR) in the rat has been reported by Haberly \& Price, ${ }^{18}$ though no topographic organization was discussed. As shown schematically in Fig. 7A, we found that while pyramidal neurons throughout the entire length of AIp and PR project to AIv, only those in the anterior two-thirds of Alp project to AId. The known inputs to AIp and PR in rodents are not topographically arranged; the anterior cortical, lateral, and anterior basolateral amygdaloid nuclei all project to the full extent of AIp and PR. ${ }^{30.36}$

In the coronal plane, the level of the genu of the corpus callosum can be regarded as the point at which AIp replaces AIv and AId, since this is the same level at which labelled cells are no longer seen in contralateral agranular insular cortex (commissural projection) and begin appearing in ipsilateral agranular insular cortex (AIp). This boundary is indistinct cytoarchitectonically but is also defined by the pattern of other connections of AIp. ${ }^{35.36}$

\section{Thalamocortical connections}

Our results confirm previous findings that AId and AIv each receive their major thalamic input from MD. ${ }^{12,13,17,35,38}$ Krettek \& Price ${ }^{35}$ found that fibers from MD terminate in layer III and outer layer I of the cortex. The terminology of Krettek \& Price $^{35}$ is used below to denote subdivisions of MD and it takes account of the differing terminology of Leonard ${ }^{38}$ and Heimer. $^{21}$

Whereas our experiments have demonstrated that the anterior portion of the medial segment of MD (MDma) projects to AIv (see Fig. 8), the results of anterograde and retrograde tracing studies in the rat indicate that $\mathrm{AIV}$ receives its major input from the central segment of $\mathrm{MD}, \mathrm{MDc}^{17,35,38} \mathrm{We}$ refer this discrepancy to the fact that in the hamster there is no well-defined central segment of MD, either in Nisslstained or myelin-stained sections. In the rat a central segment is difficult to define in Nissl-stained material but appears as a fiber-rich zone in myelin-stained material. ${ }^{35}$ Since it has been reported that the rat MDc receives olfactory input from primary olfactory cortex, endopiriform nucleus and olfactory tubercle $^{21,36.48,58,60}$ and projects to AIv, it is natural to wonder if in the hamster, MDma, which projects to AIv, is the recipient of olfactory input. If so, it may be that a presumptive central segment of MD is contained within MDma in the hamster. According to Krettek \& Price, ${ }^{35}$ the only cortical projection of 
MDma in the rat is to the prelimbic area of medial frontal cortex

There is general agreement that in both the rat and hamster, Ald receives its major thalamic input from the posterior portion of the medial segment of MD, MDmp (our results, Krettek \& Price ${ }^{35}$ Leonard. $^{38}$ ) In their HRP study in the rat, Gerfen \& Clavier ${ }^{17}$ found that the boundary area between MDmp and the parafascicular nucleus contained the greatest number of labelled cells following deposits into AId. This is consistent with our observation that labelled cells in MDmp are contiguous with those in the parafascicular nucleus, often within the same section. Other workers have also reported a projection from the parafascicular nucleus to AId in the rat (experiment R4 of Jones \& Leavitt ${ }^{2 \hbar}$, Krettek \& Price $^{35}$ ). Our results indicate that AIv also receives input from the parafascicular nucleus in the hamster. Based on cytoarchitectural criteria and on its widespread connections with various cortical areas. the parafascicular nucleus has been treated as the posterior portion of the intralaminar group of thalamic nuclei, with the central medial, paracentral and central lateral nuclei comprising the anterior portion. ${ }^{26}$ The central medial nucleus, like the parafascicular, projects to both Ald and AIV in the hamster (our results) and the rat. ${ }^{17.26}$

Our finding that the gelatinosus nucleus projects to both AId and AIv, but more heavily to the latter, agrees with the report of Gerfen \& Clavier ${ }^{17}$ and with a conclusion drawn by Herkenham ${ }^{23}$ in his study of the connections of the ventromedial thalamic nucleus.

\section{Olfactory projections to AIV}

Several areas known to receive direct input from the main olfactory bulb, namely posterior primary olfactory cortex, lateral entorhinal cortex and the posterolateral cortical nucleus of the amygdala, $C 2^{10,57}$ were found in the present study to project to Alv. The projection from $\mathrm{C} 2$ to AIv has been confirmed autoradiographically in the hamster by Kevetter \& Winans. ${ }^{30}$ Other workers have reported similar olfactory projections to $\mathrm{Alv}$ in the rat, from posterior primary olfactory cortex ${ }^{17,18.36}$ and from $\mathrm{C} 22^{36}$ Fibers from these olfactory areas terminate in layer I of AIv. ${ }^{30,3 n}$

Ours is the first report of a projection from lateral entorhinal cortex to AIv, and this is reciprocated by a projection from AIv to lateral entorhinal cortex ${ }^{49}$ Some of our HRP deposits in AIV involved the border zone between AIv and anterior primary olfactory cortex. It is therefore important to question whether encroachment on the anterior primary olfactory cortex resulted in labelled cells in lateral entorhinal cortex, which like the former is a tertiary olfactory area. First, deposits in AIv which did not involve this border zone did produce labelled cells in lateral entorhinal cortex. Second, Haberly \& Price $^{18}$ reported that in the rat, lateral entorhinal cortex projects only to medial areas of the olfactory forebrain and did not find labelled cells in lateral entorhinal cortex follow- ing HRP injections into anterior primary olfactory cortex. Therefore, it appears that lateral entorhinal cortex projects to AIv but not to anterior primary olfactory cortex, in both hamsters and rats.

Our finding that the projection from posterior primary olfactory cortex to Alv originates mostly $(65 \%)$ from layer III cells and less so $(35 \%)$ from layer IIb cells is in accord with the results of Haberly \& Price. ${ }^{18}$

Considering the demonstrated anatomical separation between the main and accessory (vomeronasal) olfactory systems ${ }^{10.57}$ and the probable differences in function of these systems (see, for example, Johns ${ }^{25}$, Wysocki ${ }^{67}$ ), it is notable that none of the tertiary accessory olfactory areas (medial and posteromedial cortical nuclei of the amygdala, bed nucleus of the stria terminalis) were found to project to AIV, either in our study or in the autoradiographic study of Kevetter \& Winans, ${ }^{29}$ also in the hamster.

\section{Amygdala projections to AId}

Previous reports are consistent with our finding of a substantial projection to Ald from the anterior portion of the basolateral nucleus. ${ }^{17,36}$ Fibers from BLa terminate in two bands within Ald, a heavy one in layer $\mathrm{V}$ and a lighter one in layers $I$ and II. $^{36}$

In the rat, reciprocal connections have been reported to exist between BLa and MDmp. ${ }^{35,36}$ both of which project to AId in hamsters (our results) and rats. $^{35,36}$ In a recent HRP study, Otterson \& Ben$\mathrm{Ari}^{40}$ found that thalamic projections to BLa originate in the interanteromedial, paraventricular and parataenial thalamic nuclei but not from MD. Similarly, the autoradiographic study of Turner \& Herkenham $^{63}$ indicates that thalamic input to $\mathrm{BLa}$ is from the interanteromedial, central medial and parataenial nuclei. As Otterson \& Ben-Ari ${ }^{46}$ suggest, it is likely that Krettek \& Price's injections into MD encroached upon these nearby nuclei, thereby producing terminal field labelling in BLa.

The subcortical projections of BLa are directed to the ventral striatum, ventral pallidum and hypothalamus, ${ }^{37.43 .44}$ which are major output pathways of the limbic system.

The presence of labelled cells in the lateral nucleus of the amygdala after AId deposits is consistent with Krettek \& Price's ${ }^{36}$ finding that the cortical projection field of the lateral nucleus appears to overlap with that of BL in the rat. Similarly, the results of Otterson $\&$ Ben-Ari ${ }^{46}$ and Otterson ${ }^{45}$ indicate a high degree of overlap in the sources of input to the lateral and basolateral nuclei. In cats there is a more distinct separation between the efferent cortical projections of these two nuclei, ${ }^{36}$ indicating either a greater separation of the cortical fields or of the lateral and basolateral nuclei.

The ventral caudal diencephalon and dopaminergic: input to Ald

Several reports $5,12,17,40.47$ have indicated the exist- 

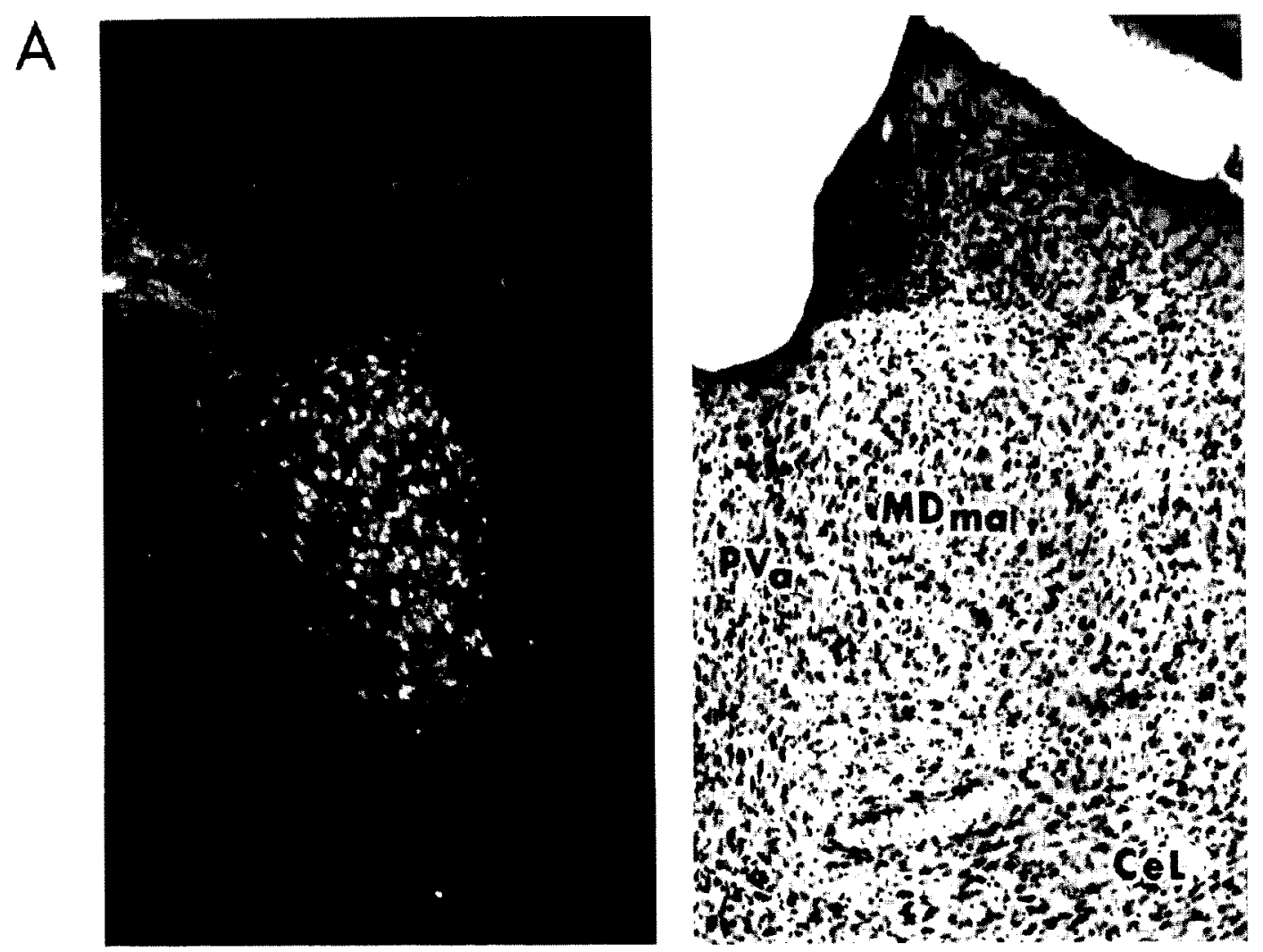

B
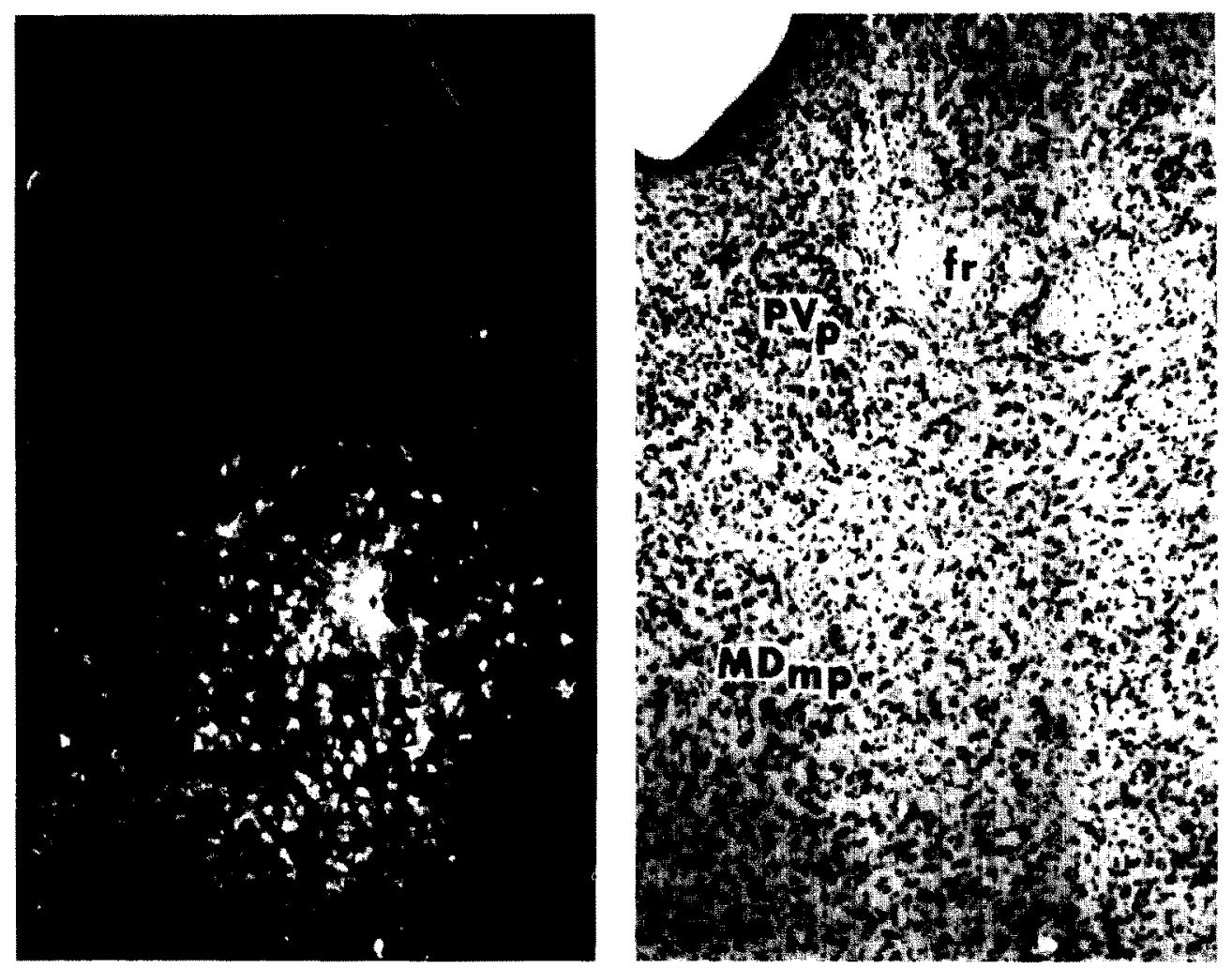

Fig. 8 . Labeled cells in the mediodersal thatamic nueleus after a horseradish peroxidase deposit which affected dorsal and ventral agranulitr insular cortex. Sections in $\mathrm{A}$ (at the same level as in Fig. 5, HI are more anterior than those in $\mathbf{B}$ tat the same level as in $\Gamma \mathrm{g}$. 3.K). Dark-field photomicrographs of TMB-reacted sections are on the left, and bright-feld photomicrographs of adjacent Cresyl Violet 
ence of dopaminergic input to AId in the rat, originating from cells in the anterior portion of the ventral tegmental area, or area A10 in the terminology of Dahlström \& Fuxe. ${ }^{9}$ In the hamster, we have found projections to AId from the lateral and posterior hypothalamus. Most labelled cells in this area are found in a region which appears to correspond to area A11, a zone shown by Bjorklund \& Nobin ${ }^{6}$ to contain catecholaminergic neurons in rats. The topographic difference between rats and hamsters regarding the source of input to AId from the ventral caudal diencephalon is paralleled by the topographic pattern of input from dopaminergic areas to nucleus accumbens. This nucleus receives input from areas A8, A9 and $\mathrm{A} 10$ in rats, but only from A10 in hamsters. ${ }^{43} \mathrm{As}$ suggested by Newman and Winans, there may be "differences in the topographic organization of the dopaminergic 'nucleus' in these two species."

In the absence of histofluorescence studies in the hamster, our results are merely suggestive of dopaminergic input to AId from the ventral caudal diencephalon.

\section{Brainstem projections to $A I d$ and $A I v$}

The dorsal raphe nucleus projects to AId and AIv in both hamsters (our results) and rats. ${ }^{17}$ This nucleus is the source of widespread serotonergic projections in rats $^{64}$ and may be in hamsters also.

Noradrenergic input to AId and AIv originates from locus coeruleus in rats. ${ }^{17,39}$ We have rarely seen labelled cells in locus coeruleus in the hamster. Perhaps because of the profuse branching which occurs in noradrenergic neurons projecting to the cerebral cortex, ${ }^{39}$ HRP is largely spread throughout the axonal branches rather than being transported posteriorly to the cells of origin. Alternatively, noradrenergic axon terminals may not uptake HRP as efficiently as non-noradrenergic terminals.

Our results indicate that the medial and lateral parabrachial nuclei project to AId in the hamster. Likewise, we have found a reciprocal projection from AId to both divisions of the parabrachial nucleus. ${ }^{49}$ In their study of the efferent projections of the parabrachial nucleus in the rat, Saper \& Loewy ${ }^{55}$ found projections from the medial parabrachial nucleus to infralimbic cortex medially and granular insular (taste) cortex laterally (to layers $\mathrm{V}$ and VI in the latter). According to their Fig. 4A, terminals are present in the anterior portion of AIp as well. It may be that in the hamster, input to AI from the parabrachial nucleus is directed to an area that includes posterior Ald and anterior AIp.

\section{Conclusions and possible functional implications}

The use of the term 'limbic lobe' from the time of Broca onwards has referred to brain regions which surround the medial border of the hemisphere, adjacent to the foramen of Monro, and to scveral cortical areas which form an outer ring around these central zones and constitute the limbic cortex. ${ }^{65}$ Although many earlier anatomists (e.g. Elliot Smith) and some later ones (e.g. Sanides, ${ }^{54}$ White $^{65}$ Yakovlev $^{68}$ ) have understood the ring-like nature of limbic cortex and the fact that it includes orbital and insular, as well as medial areas, the term 'limbic cortex' has commonly been applied only to the medially-located cingulate, retrosplenial and presubicular cortical areas, probably because of their connections with the anterior thalamic nuclei (see, for example, Robertson \& $\mathrm{Kaitz}^{50}$ ) and the inclusion of the latter in the well known Papez circuit of the limbic system. The studies of Leonard, ${ }^{38}$ Domesick, ${ }^{14}$ Beckstead $^{3}$ and Krettek \& Price ${ }^{35}$ have shown that projections from the anterior and mediodorsal thalamic nuclei overlap in the anterior cingulate cortex, causing the realization that MD projects to limbic cortex as it is commonly defined. The ring of cortex understood by earlier anatomists to constitute limbic cortex includes other areas which are known to receive MD input, namely medial frontal, orbital frontal and agranular insular areas. While the cortical projection field of MD is usually thought of as prefrontal cortex, we think it is more accurate to view it as limbic cortex in animals such as rodents, which have no granular prefrontal area. Furthermore, the projection from archicortex (pre- and parasubiculum) to the anterior thalamic nuclei, ${ }^{62}$ and the projection of the latter to medial limbic cortex ${ }^{3.14}$ is paralleled by a projection from paleocortex (primary olfactory cortex) to the mediodorsal thalamic nucleus, and the projection of the latter to agranular insular cortex (see Thalamocortical connections above). Thus, both these circuits involve projections from allocortex primitivus to the thalamus, and from there to periallocortical and proisocortical areas. The MD cortical projection fields can also be viewed as periallocortical and proisocortical portions of an agranular cortical belt which includes precentral isocortex as well. This agranular belt was first recognized on cytoarchitectural grounds by von Economo and was later shown by $\mathrm{Kaada}^{27}$ to possess functional continuity as well.

Behavioral studies in hamsters and rats have shown that lesions of agranular insular cortex, which typically involve both AId and AIv, produce dramatic changes in several species-typical behaviors, most notably those cued by olfactory stimuli. Eichenbaum and his co-workers ${ }^{15,56}$ found that male hamsters with lesions of MD or AI have normal odor detection thresholds but show reduced attraction to conspecific female or male odors. The hamsters' odor discrimination ability was also reduced. Animals with MD or AI lesions continued to mate successfully but spent more time (relative to normal, sham-operated, or medial frontal-cortex-lesioned animals) sniffing nongenital body parts and often mounted the female in an inappropriate fashion. Similar effects on odor discrimination were seen in rats following lesions of MD or AI. These workers postulate that olfactory input to AI (via MD) influences odor discriminative aspects of odor preference and sexual performance but does not affect odor detection or the priming of sexual behav- 
ior by odor cues. Kolb and his co-workers have found that $\mathrm{AI}$ lesions in hamsters and rats result in weight loss due to transient aphagia, increased aggression, increased locomotor activity and changes in mating behavior. ${ }^{31,32.33,59}$ Increased activity has also been seen in guinea-pigs following lesions of agranular insular cor tex. ${ }^{41}$

In rats, electrodes placed in AI can mediate intracranial self-stimulation, ${ }^{34.53 \text { a }}$ a response usually associated with motivation and reward. Injection of 6-hydroxydopamine into the mesocortical dopamine fibers (from A10) abolishes this response. ${ }^{8}$ and it is attenuated by systemic injection of apomorphine, a dopamine receptor agonist. ${ }^{42}$ Similarly, electrolytic lesions of AI abolish intracranial self-stimulation obtained from electrodes in $\mathrm{A} 100^{7}$ These findings strongly imply that the dopaminergic pathway from A10 to AId plays a major role in the ability of AI to support intracranial self-stimulation. By implication this suggests that AId is a site where a rewarding or motivational bias is conferred either upon inputs to Ald or outputs from it.

We suspect that by virtue of its extensive olfactory connections, Alv serves as an interface between olfactory discrimination and related behaviors, while AId. due to its connections with the basolateral amygdala and dopaminergic centers, mediates the influence of affective biasing on non-olfactory behaviors. Behavioral studies utilizing discrete lesions restricted to AId and AIv could determine whether this is the case.

Acknowledgements-We thank L. L. Reep for her artistic contributions and $\mathrm{B}$. Johnson for typing the manuscript. This research was supported by $\mathrm{NIH}$ postdoctoral grant NS-06060 to Dr Reep and by NINCDS grant NS-14071 to Dr Winans.

\section{REFERENCES}

1. Abbie A. A. (1940) Cortical lamination in the Monotremata. J. comp. Neurol. 72, 428-467.

2. Abbie A. A. (1942) Cortical lamination in a polyprotodont marsupial, Parameles nasuta. J. comp. Neurol. 76, $509-536$.

3. Beckstead R. M. (1976) Convergent thalamic and mesencephalic projections to the anterior medial cortex in the rat. $J$. comp. Neurol. 166, 403-416.

4. Beckstead R. M. (1979) An autoradiographic examination of corticocortical and subcortical projections of the medio-

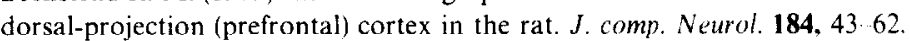

5. Berger B., Thierry A. M.. Tassin J. P. \& Moyne M. A. (1976) Dopaminergic innervation of the rat prefrontal cortex: a flourescence histochemical study. Brain Res. 106, $133-145$.

6. Björklund A. \& Nobin A (1973) Flourescence histochemical and microspectro-flourometric mapping of dopamine and noradrenaline cell groups in the rat diencephalon. Brain Res. 51, 193-205.

7. Clavier R. M. \& Corcoran M. E. (1976) Attenuation of self-stimulation from substantia nigra but not dorsal tegmental noradrenergic bundle by lesions of sulcal prefrontal cortex. Brain Res. 113, 59-69.

8. Clavier R. M. \& Gerfen C. R. (1979) Self-stimulation of the sulcal prefrontal cortex in the rat: direct evidence for ascending dopamine mediation. Neuroscience Letters 12, 183-187.

9. Dahlström A. \& Fuxe K. (1964) Evidence for the existence of monoamine-containing neurons in the central nervous system-I. Demonstration of monoamines in the cell bodies of brain stem neurons. Acra physiol. scand. 62, Suppl. 232, $1-55$.

10. Davis B. J., Macrides F., Youngs W. M., Schneider S. P. \& Rosene D. L. (1978) Efferents and centrifugal afferents of the main and accessory olfactory bulbs in the hamster. Brain Res. Bull. 3, 59-72.

11. de Olmos J., Hardy H. \& Heimer L. (1978) The afferent connections of the main and the accessory olfactory bulb formations in the rat: an experimental HRP-study. J. comp. Neurol. 181, 213-244.

12. Divac I., Björklund A., Lindvall O.\& Passingham R. E. (1978) Converging projections from the mediodorsal thalamic nucleus and mesencephalic dopaminergic neurons to the neocortex in three species. J. comp. Neurol. 180, 59-72.

13. Divac I., Kosmal A., Björklund A. \& Lindvall O. (1978) Subcortical projections to the prefrontal cortex in the rat as revealed by the horseradish peroxidase technique. Neuroscience 3, 785-796.

14. Domesick V. B. (1972) Thalamic relationships of medial cortex in the rat. Brain Behav. Evol. 6, 457-483.

15. Eichenbaum II., Shedlack K. J. \& Eckmann K. W. (1980) Thalamocortical mechanisms in odor-guided behavior--I. Effects of lesions of the mediodorsal thalamic nucleus and frontal cortex on olfactory discrimination in the rat. Brain Behav. Evol. 17, 255-275.

16. Filiminoff I. N. (1947) A rational subdivision of the cerebral cortex. Arch. Neurol. Psychiat. 58, 296-311.

17. Gerfen C. R. \& Clavier R. M. (1979) Neural inputs to the prefrontal agranular insular cortex in the rat: Horseradish peroxidase study. Brain Res. Bull. 4, 347-353.

18. Haberly L. B. \& Price J. L. (1978) Association and commissural fiber systems of the olfactory cortex in the rat 1 . Systems originating in the piriform cortex and adjacent areas. J. comp. Neurol, 178, 711-740.

19. Haberly L. B. \& Price J. L. (1978) Association and commissural fiber systems of the olfactory cortex in the rat. - II. Systems originating in the olfactory peduncle. J. comp. Neurol. 181, 781808.

20. Haug F.-M. S. (1976) Sulphide silver pattern and cytoarchitectonics of parahippocampal areas in the rat. Adv. Anat. Embryol. Cell Biol. 52, 1-73.

21. Heimer L. (1972) The olfactory connections of the diencephalon in the rat. Brain Behav. Evol. 6, 484-523.

22. Heimer L. (1978) The olfactory cortex and the ventral striatum. In Limbic Mechanisms: The Continuing Evolution of the Limbic System Concept (eds Livingston K. E. \& Hornykiewicz O.) pp. 95-187. Plenum Press, New York. 
23. Herkenham M. (1979) The afferent and efferent connections of the ventromedial thalamic nucleus in the rat. $J$. comp. Neurol. 183, 487-518.

24. Hicks S. P. \& D'Amato C. J. (1968) Cell migrations to the isocortex in the rat. Anat. Rec. 160, 619-634.

25. Johns M. A. (1980) The role of the vomeronasal system in mammalian reproductive physiology. In Chemical Signals. Vertebrates and Aquatic Invertebrates (eds Muller-Schwarze D. \& Silverstein R. M.) pp. 341-364. Plenum Press, New York.

26. Jones E. G. \& Leavit R. Y. (1974) Retrograde axonal transport and the demonstration of non-specific projections to the cerebral cortex and striatum from thalamic intralaminar nuclei in the rat, cat and monkey. J. comp. Neurol. 154, 349-378.

27. Kaada B. R. (1951) Somato-motor, autonomic and electrocorticographic responses to electrical stimulation of 'rhinencephalic' and other structures in primates, cat and dog. Acta physiol. scand. 24, Suppl. 83, 1-285.

28. Kaada B. R. (1960) Cingulate, posterior orbital, anterior insular and temporal pole cortex. In Handbook of Physiology, Section 1: Neurophsiology (eds Field J.. Magoun H. W. \& Hall V. E.) Vol. 2, pp. 1345-1372. American Physiological Society, Washington, D. C.

29. Kevetter G. A. \& Winans S. S. (1981) Connections of the corticomedial amygdala in the golden hamster-1. Efferents of the 'vomeronasal amygdala.' J. comp. Neurol. 197, 81-98.

30. Kevetter G. A. \& Winans S. S. (1981) Connections of the corticomedial amydala in the golden hamster-II. Efferents of the 'olfactory amygdala.' J. comp. Neurol. 197, 99-112.

31. Kolb B. (1974a) Dissociation of the effects of lesions of the orbital or medial aspect of the prefrontal cortex of the rat with respect to activity. Behav, Biol, 10, 329-343.

32. Kolb B. (1974b) Social behavior of rats with chronic prefrontal lesions. J. comp. Physiol. Psych. 87, 466-474.

33. Kolb B., Whishaw I. Q. \& Schallert T. (1977) Aphagia, behavior sequencing and body weight set point following orbital frontal lesions in rats. Physiol. Behav. 19, 93-103.

34. Koolhaus J. M., Mora F. \& Phillips A. G. (1977) Effects of food and water deprivation on self-stimulation of the medial and sulcal prefrontal cortex and caudate putamen in the rat. Physiol. Behav. 18, 329-331.

35. Krettek J. E. \& Price J. L. (1977) The cortical projections of the mediodorsal nucleus and adjacent thalamic nuclei in the rat. J. comp. Neurol. 171, 157-192.

36. Krettek J. E. \& Price J. L. (1977) Projections from the amygdaloid complex to the cerebral cortex and thalamus in the rat and cat. J. comp. Neurol. 172, 687-722.

37. Krettek J. E. \& Price J. L. (1978) Amygdaloid projections to subcortical structures within the basal forebrain and brainstem in the rat and cat. $J$. comp. Neurol. 178, 225254.

38. Leonard C. M. (1969) The prefrontal cortex of the rat--I. Cortical projection of the mediodorsal nucleus-II. Efferent connections. Brain Res. 12, 321-343.

39. Levitt P. \& Moore R. Y. (1978) Noradrenaline innervation of the neocortex in the rat. Brain Res. 139, 219-231.

40. Lindvall O., Björklund A. \& Divac I. (1978) Organization of catecholamine neurons projecting to the frontal cortex in the rat. Brain Res. 142, 1-24.

41. Markowitsch H. J., Guldin W., Kessler J. \& Riess R. (1980) Activity changes following sulcal, but not medial, ablation of the prefrontal cortex of the guinea-pig. Physiol. Psych. 8, 320-324.

42. Mora F., Phillips A. G., Koolhaus J. M. \& Rolls E. T. (1976) Prefrontal cortex and neostriatum self-stimulation in the rat: differential effects produced by apomorphinc. Brain Res. Bull. 1, 421-424.

43. Newman R. \& Winans S. (1980) An experimental study of the ventral striatum of the golden hamster--I. Neuronal connections of the nucleus accumbens. $J$. comp. Neurol. 191, 167-192.

44. Newman R. \& Winans S. (1980) An experimental study of the ventral striatum of the golden hamster-II. Neuronal connections of the olfactory tubercle. J. comp. Neurol. 191, 193-212.

45. Otterson O. P. (1980) Afferent connections to the amygdaloid complex of the rat and cat-II. Afferents from the hypothalamus and basal telencephalon. $J$. comp. Neurol. 194, 267-289.

46. Otterson O. P. \& Ben-Ari Y. (1979) Afferent connections to the amygdaloid complex of the rat and cat-I. Projections from the thalamus. $J$. comp. Neurol. 187, 401-424.

47. Phillipson O. T. (1979) Afferent projections to the ventral tegmental area of Tsai and interfascicular nucleus: a horseradish peroxidase study in the rat. J. comp. Neurol. 187, 117-144.

48. Powell T. P. S., Cowan W. M. \& Raisman G. (1965) The central olfactory connexions. J. Anat. 99, 791-813.

49. Rccp. R. L. \& Winans S. S. (1982) Efferent comections of dorsal and ventral agranular insular cortex in the hamster, Mesocricetus auratus. Neuroscience 7 (in press).

50. Robertson R. T. \& Kaitz S. S. (1981) Thalamic connections with limbic cortex-I. Thalamocortical connections. $J$ comp. Neurol. 195, 501-525.

51. Rose M. (1928) Die Ontogenie der Inselrinde. Zugleich ein Beitrag zur histogenetischen Rindeneinteilung. J. Psychol. Neurol. 36, 182-209.

52. Rose M. (1928) Die Inselrinde des Menschen und der Tieren. J. Psychol. Neurol. 37, 467-624.

53. Rose J. E. \& Woolsey C. N. (1948) The orbitofrontal cortex and its connections with the mediodorsal nucleus in rabbit, sheep and cat. Assoc. Res. Nerv. Ment. Dis. 27, 210-232.

53a Routtenberg A. \& Sloan M. (1972) Sell stimulation in the frontal cortex of Rattus norvegicus. Behav. Biol. 7, 567-572.

54. Sanides F. (1970) Functional architecture of motor and sensory cortices in primates in light of a new concept of neocortex evolution. In The Primate Brain (eds Noback C. R. \& Montagna W.) pp. 137-203. Appleton-CenturyCrofts, New York.

55. Saper C. B. \& Loewy A. D. (1980) Efferent connections of the parabrachial nucleus in the rat. Brain Res. 197, $291-317$.

56. Sapolsky R. M. \& Eichenbaum H. (1980) Thalamocortical mechanisms in odor-guided behavior-II. Effects of lesions 
of the mediodorsal thalamic nucleus and frontal cortex on odor preference and sexual behavior in the hamster. Brain Behav. Evol. 17, 276-290.

57. Scalia F. \& Winans S. S. (1975) The differential projections of the olfactory and accessory olfactory bulb in mammals. J. comp. Neurol. $161,31-56$.

58. Scott J. W. \& Leonard C. M. (1971) The olfactory connections of the lateral hypothalamus in the rat, mouse and hamster. J. comp. Neurol, 141, 331-344.

59. Shipley J. E. \& Kolb B. (1977) Neural correlates of species typical behavior in the Syrian golden hamster. $J$. comp. Physiol. Psych. 91, 1056-1073.

60. Siegel A., Fukushima T., Meibach R., Burke L., Edinger H. \& Weiner S. (1977) The origin of the afferent supply to the mediodorsal thalamic nucleus: enhancement of HRP transport by selective lesions. Brain Res. 135, 11-23.

61. Stephan H. \& Andy O. J. (1970) The allocortex in primates. In The Primate Brain (eds Noback C. R. \& Montagna W.) pp. 109-135. Appleton-Century-Crofts, New York.

62. Swanson L. W. \& Cowan W. M. (1977) An autoradiographic study of the organization of the efferent connections of the hippocampal formation in the rat. $J$. comp. Neurol. $172,49-84$.

63. Turner B. \& Herkenham M. (1981) An autoradiographic study of thalamo-amygdaloid connections in the rat. Anat. Re'. 199, 260A.

64. Ungerstedt U. (1971) Stereotaxic mapping of the monoamine pathways in the rat brain. Acta physiol. scand. 82, Suppl. $367,1-48$.

65. White L. E. (1965) A morphological concept of the limbic lobe. Int. Rev. Neurohinl 8, 1-34.

66. Wise S, P. \& Jones E. G. (1976) The organization and postnatal development of the commissural projection of the rat somatic sensory cortex. J. comp. Neurol, 168, 313-344.

67. Wysocki C. J. (1979) Neuro-behavioral evidence for the involvement of the vomeronasal system in mammalian reproduction. Neurosci. Biobehav. Rev, 3, 301-341.

68. Yakovlev P. 1. (1948) Motility, behavior and the brain. J. Nerv. Ment. Dis. 107, 313-335. 